\title{
MOVES: a MemOry-based VEhicular Social Forwarding Technique
}

\author{
Anna Maria Vegni ${ }^{a, *}$, Carlos Borrego Iglesias ${ }^{\mathrm{b}}$, Valeria Loscríc ${ }^{\mathrm{c}}$ \\ ${ }^{a}$ Department of Engineering, Roma Tre University, Italy 4 \\ ${ }^{b}$ Department of Mathematics and Computer Science, University of Barcelona, Spain 5 \\ ${ }^{c}$ INRIA Lille-Nord Europe, France
}

\begin{abstract}
Recently, the new paradigm of Vehicular Social Networks (VSNs) has stimulated

a lot of interest in the research community. The rationale behind this interest relies on the integration of social relations in the Internet of Vehicles, taking 10 into account of the human component -in terms of preferences and interests of ${ }_{11}$ individuals-, and then allowing to distinguish nodes based on social ties. This 12 feature affects the content dissemination procedures in VSNs, so that the most ${ }_{13}$ social node within a transmission range is expected to be the most appropriate ${ }_{14}$ next-hop forwarder, for higher network performance achievement. 15

Leveraging on such premises, in this paper we propose a MemOry-based ${ }_{16}$ VEhicular Social forwarding approach, namely MOVES, that builds its packet ${ }_{17}$ forwarding logic by considering both the past and present "social" pattern of ${ }_{18}$ the nodes. MOVES is inspired by a previous forwarding mechanism, namely 19 SCARF, that integrates the social components and physical features in its for- 20 warding mechanism. MOVES has been compared to SCARF, and other existing $\quad 21$ forwarding approaches, in different scenarios and with real data in terms of de- 22 livery ratio, overhead and latency; results show its effectiveness of including the ${ }_{23}$ "social memory" in the selection of the next-hop forwarder. 24 Keywords: Vehicular social networks, packet forwarding probability, social 25 features, next-hop forwarder selection. $\quad 26$
\end{abstract}

\footnotetext{
${ }^{*}$ Corresponding author

Email addresses: annamaria.vegni@uniroma3.it (Anna Maria Vegni), carlos.borrego@ub.edu (Carlos Borrego Iglesias), valeria.loscri@inria.fr (Valeria Loscrí)

Preprint submitted to Elsevier Computer Networks

July 9, 2021
} 


\section{Introduction}

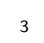

4

The concept of social networking is largely applied to different contexts, ranging from crowd-sourcing data collection to dissemination and forwarding in information networks, as well as in information and coding theory. One of the main representations of social networking are the Online Social Networks (OSNs) [1], comprised of people with social interactions and relationships, that share common interests thus forming social communities. Platforms like Facebook, Twitter and LinkedIn are used everyday by users to be in contact with friends, colleagues, public celebrities, and so on.

OSNs have recently evolved towards the concept of mobile social networks, where users are in mobility in a very dynamic environment, such as the vehicular networks. This evolution has been driven by the observation of users' behavior in terms of $(i)$ mobility pattern and (ii) social behavior. As known, due to the nature of vehicular networks, mobility and traffic patterns can provide social interactions among neighbors. As an instance, in heavy traffic scenarios, the vehicular density is very high and traffic pattern is relatively static. Such scenario becomes a popular social place for vehicles to connect to each other, and share information (e.g., traffic information, weather news, points of interest, and so on) hop-by-hop.

This evolution has carried out the concept of Vehicular Social Networks (VSNs) that consists of groups of individuals that may share common interests, preferences and needs in the context of a temporal-spatial proximity on roads $[2,3]$. VSNs are emerging as a novel communication paradigm that exploits opportunistic encounters among vehicles for mobile social networking and collaborative content dissemination. Vehicles are then members of a mobile social network, which is formed on-the-fly among neighbouring vehicles. Differently from traditional vehicular ad-hoc networks, data dissemination and routing in VSNs can exploit node social features.

The main motivations of this paper rely on the necessity to implement effective and efficient forwarding mechanisms able to account for the social fea- 
tures [4]. In practice, in a VSN beyond the requirements and constraints typical 1 of a high dynamic context as the vehicular networks, we can exploit the node 2 social relations. Then, the main challenges for achieving effective forwarding 3 mechanisms in VSNs are based not only on the social nature of the nodes, but 4 also rely on the "correct" definition of sociality of the nodes. How to define their 5 role in the network, to make the forwarding mechanisms more effective and to 6 keep a certain fairness among the nodes, is also a topic to investigate. 7

In particular, one of the main challenges in VSNs is the identification of 8 "the most social node" i.e., the node that exhibits more social features and 9 social behavior. The sociability of a node is a concept that needs definitions. 10 Usually, centrality metrics are used to denote how "important" a node is inside 11 a network $[5,6,7]$, and in the context of VSNs, the importance of a node 12 is referred to its sociability [8, 9]. Indeed, in a VSN a social node is usually 13 referred also as hub node, i.e., a node with the highest number of links in the $\quad 14$ whole network. Having many one-hop neighbors allows to reach many nodes 15 in case of broadcast data dissemination, thus obtaining a more effective packet $\quad 16$ forwarding $[10,11]$. 17

A definition of hub node has been proposed in [12], through the concept of ${ }_{18}$ node degree of graph theory. A hub node is then a central node, and in case 19 of social networks it can show social activity $[13,14,15,16,17,18]$. Different 20 definitions of social activity have been proposed. In [18], Aylani and Goyal ${ }_{21}$ introduce the Common Social Activity parameter, which exploits information 22 about tags and comments sent by a user. A similar approach has been presented 23 by Peleshchyshyn et al. in [17], where several indicators have been considered 24 for user social activity, such as the average frequency of content and the socially $\quad 25$ relevant content. Finally, Socialite [16] is a social activity mining framework, 26 able to achieve social group discovering by exploring users' interactions. 27

Information about node social activity results also as a criterion for next-hop $\quad 28$ forwarder selection. In [15], Li et al. consider a relay node as the one having 29 more common interests with a destination node, and also having a higher local 30 activity in the community, i.e., it shows many interactions with other members 31 
1 of the community. Similarly to previous works, in case of VSNs, the paper [12] defines the node social activity following a power-law distribution, depending on the number of messages sent by the node and the probability the node is "online" in the network. This latter aspect is typical of VSNs, where connections among nodes are built dynamically and last for a limited time period only [2].

In this paper, we aim to present a novel technique for next-hop forwarder selection in VSNs, based on a novel concept for node social degree. This parameter considers the amount of messages sent and the number of connectivity links formed by a node, both observed instantaneously and in a given time window. Indeed, we observe that the node social degree shows time dependence, as user's social behavior follows predefined patterns. For instance, a user that uses to send many messages in the community is expected to have this behavior in the future. The past and present observations of the node social activity (i.e., number of messages sent and connectivity links) allow the estimation of the node social degree. As a result, the temporal dependence of social degree affects the decisions of next-hop forwarder selection.

This paper presents MOVES (MemOry-based VEhicular Social forwarding technique) i.e., a probability-based approach for next-hop forwarder selection in VSNs, based on the concept of time-varying node social degree and physical parameters as typical of Dedicated Short Range Communications (DSRC) technology. MOVES refers to a previous approach, namely SCARF (SoCial-Aware Reliable Forwarding Technique for Vehicular Communications) [19, 12], which considers a next-hop forwarder as the farthest and most social node within a source node's transmission range. SCARF takes decision on next-hop forwarder at a given time instant, and the node social degree is computed hop-by-hop. SCARF does not consider the node social behavior from past observations, but it is limited to the present. Contrary, MOVES exploits information of social degree that the $i$-th node has exhibited in the past, and mixes with the social degree experienced by the $i$-th node in the present. As a result, the node social degree estimation is expected to be more accurate, resulting in a more effective next-hop forwarder selection. 
MOVES comes from SCARF also due to dependence on physical parameters 1 (i.e., vehicles' inter-distance) that limit the next-hop selection. Specifically, 2 MOVES splits $(i)$ social features and $(i i)$ physical parameters that exist in 3 vehicular networks, as two independent events, and considers $(i)$ the probability $\quad 4$ that the social degree of the $i$-th node is the highest in a given space, and $(i i) \quad 5$ the probability that the $i$-th node is the farthest from a source node, within 6 a transmission range. The two events are statistically independent, and the 7 MOVES probability assignment function can be expressed as simply the product of the two probabilities.

In details, the following contributions are addressed in this paper:

1. We introduce a new definition for node social degree, based on both present and past observations. Specifically, we consider the ability of a node to be in connectivity with neighbors (i.e., number of connections) and the number of messages exchanged in a time window. These features allow to obtain an estimation of the node social degree, taking into account both its present and past social behavior. The rationale behind this approach is to observe how "social" a node is (and has been). Information about past and present estimations are accordingly weighted based on a given observation time period;

2. MOVES technique exploits $(i)$ the proposed concept of node social degree, as well as $(i i)$ physical features that need to be considered for an effective data transmission. Specifically, MOVES considers the probabili- 22 ties that two events may occur i.e., $(i)$ the social degree of the $i$-th node $\quad{ }_{23}$ is not lower than the social degrees of neighboring vehicles, and $(i i)$ the $\quad 24$ $i$-th node is the farthest node within a given transmission range. The two ${ }_{25}$ events are statistically independent and take into account both the social 26 aspects and the physical features, respectively. Furthermore, due to the ${ }_{27}$ time dependence of social degree, MOVES technique will be also depen- 28 dent on the past and present estimation of social degree. Leveraging on 29 such consideration, the proposed approach is a memory-based forwarding $\quad 30$ 
technique i.e., for each node, the information of past social behavior is taken into account;

3. MOVES has been assessed in real traffic scenarios and compared to other existing related approaches i.e., Epidemic, SCARF and an optimal stoppingbased technique [20]. Results are expressed in terms of $(i)$ delivery ratio, (ii) overhead, (iii) latency, and ( $i v$ ) computational cost (i.e., number of hops). We observe that MOVES outperforms other techniques for what concerns the delivery ratio, the overhead and latency, but it needs a higher number of hops to reach a final destination.

This paper is organised as follows. Section 2 deals with current related works about data dissemination techniques in VSNs, with special highlights on social-based techniques. Specifically, more attention will be given to a previous technique i.e., SCARF [19], that considers packet retransmissions based on both vehicles social degree and physical parameters. We then highlight the main differences that exist between our proposed MOVES approach and existing techniques. Section 3 presents MOVES forwarding technique via a mathematical model for next-hop forwarder selection, and presents the analysis on the average number of "social forwarder nodes". Simulation results are then carried out in Section 4, where real traffic traces have been considered for a more realistic performance assessment. A comparison to other forwarding approaches has been investigated as well. Finally, conclusions are drawn at the end of this paper.

\section{Related works}

Due to increasing popularity of OSNs and the increasing number of interconnected mobile devices by the means of the Internet of Things (IoT) paradigm, social relations and social ties have started to be studied and deeply analyzed into wireless networks. This paved the way for mobile social networks $[21,22]$ and opportunistic social networks $[23,24,25]$. The vehicular social network belong to the opportunistic social networks category. 
We identify two main related topics for our approach MOVES i.e., $(i)$ how 1 the social ties are established and used, and $(i i)$ the dissemination mech- 2 anisms. Content dissemination techniques consider how to distribute contents 3 to users or towards areas of interest. Different approaches are based on major 4 social features, such as centrality metrics [4]. One of the initial contributions on 5 the application of social relations in the vehicular context has been introduced 6 in [26], where the authors analyze the centrality metrics in vehicular ad hoc 7 networks. In particular, the authors motivate their research by considering the 8 social networks analysis (SNA) and the social structures that are formed, and 9 notice that there are similarities between sensors/vehicle networks and social 10 networks. In [27], social metrics and SNA are exploited in order to improve the 11 coverage of service channels (SCHs). It is investigated how the social metrics 12 can be applied to improve the performance of the channel occupation, while 13 SNA is considered for optimal deployment of RSUs (Road Side Units) and to ${ }_{14}$ improve data communication mechanisms. The use of RSUs along the roads can 15 enhance the network performance through Vehicle-to-Infrastructure connectiv- 16 ity links, as presented by Bitaghsir et al. in [28], where the authors introduce a ${ }_{17}$ content distribution technique based on the idea of caching content to RSUs. 18

The importance on how the social relations are defined and their impact on $\quad 19$ the network performance, also in terms of radio coverage, have been addressed 20 in [29], where the authors highlight how the weak ties are of paramount im- ${ }_{21}$ portance in order to maximize the probability of reaching more destinations. 22 In [30], Pianese et al. consider temporal, spatial and activity profiles in order to ${ }_{23}$ characterize the social relations of the users. In [31], Gong et al. consider the 24 social contribution of the relay node, expressed as the amount of $(i)$ forward- $\quad 25$ ing services each node has done to another, and viceversa, and (ii) forwarding 26 services the node provides to other nodes within the community. Gai et al. [32] 27 characterize the social relations based on reputation of a node rated by others $\quad 28$ during past transactions. A central server is considered to ensure the integrality 29 of the trust information. A Ratee-based Trust Management is built in order to 30 exploit the relationships between the nodes for increasing the trustworthiness 31 
among the nodes. In [33], Basta et al. provide a novel definition of social tie strength that characterizes the meeting frequency of wireless nodes in a vehicular context. Finally, in [19], the authors introduce a new definition of social node to be integrated in a data forwarding mechanism in a vehicular context.

The main motivation behind this paper relies on the assumption that a social node is expected to frequently meets other nodes and then is a better candidate to disseminate messages. Similar considerations have been adopted by Yao et al. in [34], which propose a cooperative caching scheme based on social attributes and mobility prediction for Vehicular Content Centric Networks. The authors assume a caching node sharing more social attributes with the other nodes is more likely to be interested in the same contents and is willing to distribute the contents to other nodes with similar interests. Also, Ullah et al. [35] exploit the computation of tie strength based on social metrics, in order to estimate node's reputation. This aspect is then assumed as main criterion for message dissemination in 5G-based vehicular networks. All the approaches considered so far demonstrate the effectiveness of including the social components for improving the performance of vehicular networks.

Content dissemination is extremely important for mobile and opportunistic social networks. In [36], in the context of opportunistic social networks, three different categories of dissemination techniques have been considered, i.e., ( $i$ ) epidemic, (ii) probability-based, and ( $i i i)$ interest community-based content dissemination. This classification matches also very well into VSNs. Different contributions belonging to the three different categories have been proposed recently. Epidemic approaches have been considered in [37], where the PeopleNet architecture relies on an already pre-existing infrastructure in order to "epidemically" send data in a mobile social network context. A hybrid approach, namely EpSoc, utilizes the Epidemic routing forwarding strategy together with social features, such as degree centrality [38]. In [39], Lee et al. propose a kind of virtual flea market based on users' interests, namely FleaNet, where the customers can express their requests and send their queries, as well as their offers, etc. Similarly, the Optimal Stopping (OS) approach [20] is a kind of message 
broadcasting technique, where forwarding nodes are selected based on their $(i) \quad 1$ centrality, (ii) reliability, and ( $i i i)$ similarity. The goal is to make the broadcast 2 effective and pervasive all over the network. 3

In [40], Priority Based Efficient Information Dissemination Protocol (PBEID) 4 has been proposed by combining probability-based and density-based infor- $\quad 5$ mation dissemination techniques with conventional broadcasting. In [41], Zhou 6 et al. regard to the interests matching among vehicular users, and introduce 7 the concept of social relationship tightness to realize the matches among users. 8 Specifically, the authors a social big data-based content dissemination approach 9 by relying on D2D (Device-to-Device) - V2V (Vehicle-to-Vehicle) connections. $\quad{ }_{10}$

A taxonomy for content dissemination in VSNs has been proposed in [42], 11 where Mezghani et al. classify the dissemination approaches based on $(i)$ infor- 12 mation processing, $(i i)$ content delivery, and $(i i i)$ performance. It is highlighted 13 that the best approach in the context of social networks is represented by the 14 class considering user preferences, even though this needs to exchange prefer- $\quad 15$ ences before sending data. In particular, the authors also consider the user 16 satisfaction as new feature to be considered in order to improve data dissem- 17 ination. Finally, the results obtained by Li et al. in [36] have highlighted a 18 deep analysis on the network topology, and it resulted that the temporal and 19 spatial characteristics have to be analyzed and integrated in the content dissem- $\quad 20$ ination schemes, for the enhancement of network performance and effectiveness 21 of the dissemination in opportunistic mobile social networks. Based on these 22 results in the context of opportunistic social networks, in this paper we consider ${ }_{23}$ the sociality of a node as a prominent feature for being considered in a data ${ }_{24}$ forwarding process.

\section{MOVES Forwarding Technique 26}

MOVES technique exploits not only physical features of the communication $\quad 27$ environment, such as node inter-distance, channel estimation, vehicular density 28 and so on, but also social features like node centrality, betweenness, degree, ${ }_{29}$ 


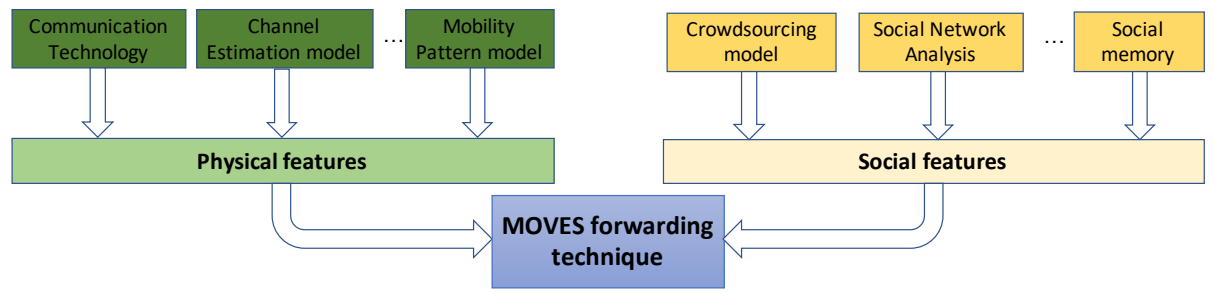

Figure 1: Main factors affecting MOVES forwarding technique.

etc. The joint combination of physical and social features allows to select the most social node within a given area as next-hop forwarder. Figure 1 depicts the main inputs affecting MOVES node selection. External factors coming from the network environment (e.g., channel estimation, communication technology, mobility pattern model, etc.) represent the physical features, while social features identify how much nodes are social and depend on graph theory metrics like centrality, betweenness, etc. By varying physical and/or social features, we can obtain different MOVES forwarding probabilities that better fit in a given network scenario. For instance, changing the definition of social memory or the wireless carrier, the resulting forwarding probability will be different. This allows to extend MOVES approach according to different requirements and needs.

MOVES main goal is to identify which node in a VSN presents the highest degree of sociality i.e., the node the most "social" in the network, since it is expected to be likely the most appropriate next-hop forwarder. As similar to SCARF [12], MOVES is applicable to main VSN applications, from security to entertainment-based ones, as it aims to select only those social vehicles likely to be the most suitable next-hop forwarders.

How sociality affects the message retransmission has been already addressed in previous works, but how to define the sociality degree of a node is a tricky issue that can be solved in several manners. In this paper, we consider the node sociality as the skill of a node of $(i)$ connecting and (ii) communicating to neighboring nodes (i.e., one-hop nodes). A node with a high node degree that 3 communicates via broadcast is then expected to exhibit a good node sociality, 
while a node with low degree and low number of packet transmissions has likely a bad sociality.

We can observe that a node is showing a good node sociality in a given time interval, and then represents a good candidate for next-hop forwarder, but this behavior is time-limited and may change quickly. For instance, let us consider a node is in contact and communicates with its neighbors for all the time interval the node is in mobility. However, due to quick variations of mobility pattern and connectivity that use to occur in vehicular environments, such node can show a totally different node sociality due to lack of neighbors and no packet to retransmit. It can be observed that the node sociality is time dependent according to both $(i)$ present and $(i i)$ past interactions with other nodes. For instance, a node that in the last $n$ days has been connected with a high number of neighbors and has sent/received multiple messages, it is mostly likely to have a similar behavior in the future. In contrast, a node that in the last $n$ days has being connected with a low number of nodes and also exchanged less messages, it is expected to have a poor skill of communicating and connecting with neighbors. It follows that its social skill is expected to be lower.

Leveraging the above considerations, we can define the social degree of the 18 $i$-th node as:

$$
s_{i}(t)=\mathcal{M}_{i}(t)+\mathcal{C}_{i}(t)
$$

where $\mathcal{M}_{i}(t)$ and $\mathcal{C}_{i}(t)$ are the time-dependent message and connection coef- $\quad 20$ ficients associated to the $i$-th node, respectively. We observe that the social 21 degree of the $i$-th node is the contribution of $(i)$ the communication degree and 22 (ii) the connectivity degree of the $i$-th node, obtained both from recent and 23 past estimations. In Eq. (1), the first term on the right of i.e., $\mathcal{M}_{i}$, represents ${ }_{24}$ the communication degree of the $i$-th node $i$.e., how many messages a node has 25 exchanged on average in the network, computed by weighting the average num- 26 ber of messages transmitted $(i)$ in a given time interval i.e., $t=\Delta T$, and $(i i) \quad{ }_{27}$ in the whole time period ${ }^{1}$, i.e., $t=T$. Specifically, we pose $\overline{\mathcal{M}}_{i}$ as the average $\quad 28$

\footnotetext{
${ }^{1}$ The parameter $T$ represents the whole time period of mobility pattern of the $i$-th vehicle
} 
1 number of messages that the $i$-th node has exchanged in the network in a given time window $t=\Delta T$, and $\overline{\mathcal{M}}_{i, h}$ as the average number of messages that the $i$-th node has used to exchange in the network in the whole time period, where ${ }_{4}$ the subscript $h$ means a historical value. The message coefficient $\mathcal{M}_{i}$ in Eq. (1) 5 can be then written as:

$$
\mathcal{M}_{i}(t)=\left\{\begin{array}{l}
\beta \overline{\mathcal{M}}_{i}(\Delta T)+(1-\beta) \overline{\mathcal{M}}_{i, h}, \\
\text { if } \overline{\mathcal{M}}_{i}(\Delta T)>\overline{\mathcal{M}}_{i, h} \\
(1-\beta) \overline{\mathcal{M}}_{i}(\Delta T)+\beta \overline{\mathcal{M}}_{i, h}, \\
\text { if } \overline{\mathcal{M}}_{i}(\Delta T) \leqslant \overline{\mathcal{M}}_{i, h}
\end{array}\right.
$$

where $\beta$ is a constant in the range $[0,1]$ used to give different weights to the historical information and the new one. By mixing both historical and most recent message coefficients, the node's social attitude can be observed. For instance, a node that used to send messages in the past few days represents a very active node, as compared to a node with a lower average number of sent messages in the same time period. The introduction of historical data, which can be stored in a local node cache, allows to take into account the node's past interactions. Specifically, for $\overline{\mathcal{M}}_{i, h}$ we compute the average number of messages exchanged by the $i$-th node in the network in the past $T$ period.

Similar considerations are applied to the second term on the right side of ${ }_{16}$ Eq. (1), i.e. $\mathcal{C}_{i}$, that represents the connectivity degree and can be expressed 17 as:

$$
\mathcal{C}_{i}(t)= \begin{cases}\beta \overline{\mathcal{C}}_{i}(\Delta T)+(1-\beta) \overline{\mathcal{C}}_{i, h}, & \text { if } \overline{\mathcal{C}}_{i}(\Delta T)>\overline{\mathcal{C}}_{i, h} \\ (1-\beta) \overline{\mathcal{C}}_{i}(\Delta T)+\beta \overline{\mathcal{C}}_{i, h}, & \text { if } \overline{\mathcal{C}}_{i}(\Delta T) \leqslant \overline{\mathcal{C}}_{i, h}\end{cases}
$$

where $\overline{\mathcal{C}}_{i}$ and $\overline{\mathcal{C}}_{i, h}$ represent the average number of connections that the $i$-node has established with its neighboring nodes, respectively $(i)$ in a given time window $t=\Delta T$, and $(i i)$ in the whole time period i.e., historical average. As

(e.g., assumed as 24 hours). Notice that $\Delta T \leq T$ [s]. In the following, we consider the ratio $r=\Delta T / T$, which varies for different $\Delta T[\mathrm{~s}]$. 
in the previous Eq. (2), the term $\beta$ is a weight constant in the range $[0,1] . \quad 1$

From Eqs. (2) and (3), we observe that the meaning of $\overline{\mathcal{M}}_{i}\left(\overline{\mathcal{C}}_{i}\right)$ and $\overline{\mathcal{M}}_{i, h} \quad$ 2 $\left(\overline{\mathcal{C}}_{i, h}\right)$ distinguishes according to the time window where exchanged messages 3 (connections) are counted on average. In the first case, we consider the average 4 number of messages exchanged by the $i$-th node in the time interval $\Delta T[\mathrm{~s}]$, while $\quad{ }_{5}$ in the latter the average number of messages is computed in the overall time 6 period. In Eq. (2) we observe a different expression for the message coefficient 7 according to the condition that is satisfied. Indeed, by comparing the average 8 number of messages that the $i$-th node has exchanged in $\Delta T[\mathrm{~s}]$ time interval 9 and in the overall time period $T[\mathrm{~s}]$, if the recent estimation is higher than the ${ }_{10}$ past one, then more weight is provided to the recent one. This means that, in ${ }_{11}$ the case $\overline{\mathcal{M}}_{i}(\Delta T)>\overline{\mathcal{M}}_{i, h}$, for $\beta>0.5$, the average number of messages in the ${ }_{12}$ recent time window $\Delta T$ has higher weight than the average number of messages $\quad{ }_{13}$ exchanged in the overall time period. Otherwise, in the case $\overline{\mathcal{M}}_{i}(\Delta T) \leq \overline{\mathcal{M}}_{i, h} \quad{ }_{14}$ and for the same values of $\beta$, less weight will be given to $\overline{\mathcal{M}}_{i}(\Delta T)$. Of course, ${ }_{15}$ the limit values i.e., $\beta=0$ and $\beta=1$, allow to consider only one term between $\quad 16$ $\overline{\mathcal{M}}_{i}(\Delta T)$ and $\overline{\mathcal{M}}_{i, h}$. These cases are however not suitable to consider, as the ${ }_{17}$ estimation of the social degree would lack of a missing term. Similar consider- ${ }_{18}$ ations apply to Eq. (3). In Section 4, we will investigate the role of $\beta$ in the ${ }_{19}$ social degree estimation in real traces. 20

Leveraging on previous considerations, the definition of social degree in ${ }_{21}$ Eq. (1) can be rewritten as

$$
s_{i}(t)=\bar{s}_{i, h}+\bar{s}_{i}(\Delta T)
$$

where the terms $\bar{s}_{i, h}$ and $\bar{s}_{i}(\Delta T)$ take into account both the average number ${ }_{23}$ of messages and the connections occurred for the $i$-th node in $(i)$ the whole ${ }_{24}$ past time period, and $(i i)$ the time window $\Delta T$, respectively. The term $\bar{s}_{i, h}{ }_{25}$ represents the "social memory" of the $i$-th node (i.e., if a node has been social ${ }_{26}$ in the past) and depends on $\overline{\mathcal{M}}_{i, h}$ and $\overline{\mathcal{C}}_{i, h}$. ${ }_{27}$

After introducing the social degree concept, we investigate how to consider ${ }_{28}$ this factor for data dissemination purpose in VSNs. Usually, message transmis- ${ }_{29}$ 
1 sion techniques in vehicular networks are based on physical factors only, such as distance, vehicular density, power level and so on. When considering social features in vehicular scenarios, such as the ability of a node to form connections with neighboring nodes, then message transmission techniques should include also social aspects. As already highlighted in previous sections, it has been demonstrated that the next-hop forwarder selection based on social features of a node provides better performance, as a more social node is expected to reach more nodes in case of packet forwarding. In VSNs, the next-hop forwarder selection occurs on the highest value of social degree that a node in a given transmission range can experience. Basically, the node with the highest social degree is expected to be elected as next-hop forwarder. Finally, notice that data exchange and gathering of relevant information among vehicles is guaranteed by assuming an edge computing architecture, as already described in [12].

Let us consider a VSN community as comprised of a given number $N$ of nodes. All the nodes are assumed to be forming a cluster of nodes, so that none of the $N$ nodes has a null node degree $i . e ., \delta_{i} \neq 0$ with $i=\{1,2, \ldots, N\}$. Figure 2 depicts a schematic of node degree in a vehicular network. Different clusters ( red rectangles) are formed and comprised of nodes with non-null degrees e.g., $\delta_{1}=3$, $\delta_{2}=2, \delta_{3}=3$, and $\delta_{4}=2$. Isolated nodes have a null degree and do not belong to any cluster e.g., $\delta_{5}=0$. Similar considerations apply for the other cluster with node degrees $\delta_{6,7,8}$ respectively equal to 1,2 and 1 .

A source vehicle (i.e., Tx node) has to forward a message towards a nexthop node within its transmission range i.e., $z[\mathrm{~m}]$. The $i$-th vehicle within the transmission range $z$ is at distance $d_{i}$ (i.e., $d_{i} \leq z[\mathrm{~m}]$ ). As known, the nodes' positions can be generated according to a Poisson Point Process of parameter $\rho$, which is the vehicle linear spatial density [veh/m], and the inter-vehicle spacing is exponentially distributed with mean $1 / \rho$. Then, we can assume $X_{i}$ as an independent and identically distributed (i.i.d.) random variable denoting the space between the $i$-th and the $(i+1)$-th vehicle, with the exponentially-distributed Cumulative Distribution Function (CDF), expressed as $F_{X}(x)$.

The forwarding probability of the $i$-th vehicle should be proportional to $(i)$ 


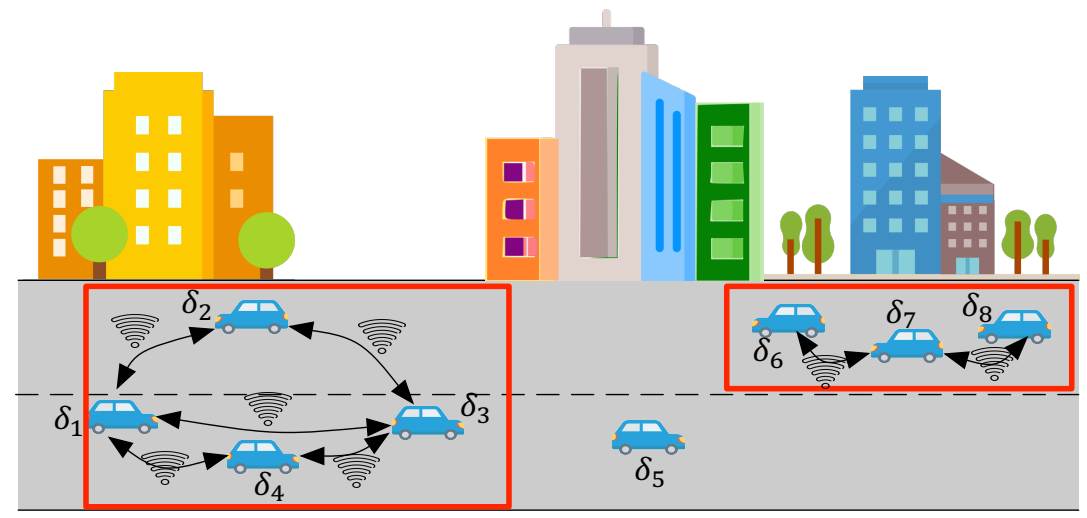

Figure 2: Schematic of node degree in a cluster (red rectangles), where nodes are in V2V communication and none of them has a null degree i.e., $\delta_{i} \neq 0$, with $i=\{1,2,3,4\}$. An isolated node is out of a cluster and has a null degree e.g., $\delta_{5}=0$. Similar considerations apply for $i=\{6,7,8\}$.

the probability that there is no vehicle in the interval of length $\left(z-d_{i}\right)[\mathrm{m}]$ able to re-forward, and (ii) the probability that there is no neighboring vehicle with a social degree higher than that of the $i$-th vehicle. The forwarding probability associated to the $i$-th node depends on two events that are independent from each other. The first event is represented by the probability that there is no other vehicle in the interval of length $\left(z-d_{i}\right)[\mathrm{m}]$, and can be expressed as

$$
p_{1, i}=1-F_{X}\left(z-d_{i}\right),
$$

where $F_{X}\left(z-d_{i}\right)$ is the CDF of $X_{i}$, with $x=z-d_{i}$. On the other side, the probability that there is no neighboring vehicle with a social degree $S$ higher than that of the $i$-th vehicle is

$$
p_{2, i}=1-\operatorname{Pr}\left\{S>s_{i}(t)\right\},
$$

where the term on the right side represents the complementary cumulative distribution function in case of Pareto distribution [43], which is a viable assumption for the social degree distribution in vehicular scenarios [12].

We can observe that Eq. (5) represents SCARF forwarding probability as- ${ }_{13}$ signment function in case of maximum value of social degree i.e., $s_{i}=1$, and ${ }_{14}$ 
1 with $c_{i} \geq 1$ as a shape factor $[12,19]$, i.e.

$$
p_{1, i}=\exp \left[-\rho \frac{z-d_{i}}{c_{i}}\right],
$$

while $p_{2, i}$ in Eq. (6) represents the complementary CDF of a Pareto random variable with parameters $\alpha$ as the exponent of the power-law distribution i.e., $\alpha=[2,3]$ for small-world networks, and $s_{m}$ is the minimum value of the social degree $s_{i}$, i.e., [43]

$$
p_{2, i}=1-\left(\frac{s_{i}(t)}{s_{m}}\right)^{(-\alpha+1)} .
$$

6 Finally, since the two events described by Eq. (7) and (8) are statistically in7 dependent, the MOVES forwarding probability assignment function of the $i$-th 8 vehicle can be expressed mathematically as:

$$
\begin{aligned}
p_{f, i}^{M O V E S}(t) & =p_{1, i} \cdot p_{2, i} \\
& =\exp \left[-\rho \frac{z-d_{i}}{c_{i}}\right] \cdot\left[1-\left(\frac{s_{i}(t)}{s_{m}}\right)^{(-\alpha+1)}\right]
\end{aligned}
$$

In Eq. (9), the selection of the $i$-th vehicle as next-hop forwarder based on social features occurs if its social degree is at least higher than a given required minimum value i.e., $s_{i} \geq s_{m}$, and no other neighboring vehicle exhibits a social degree higher i.e., $1-\operatorname{Pr}\left\{S>s_{i}(t)\right\}$. However, this condition is not enough to guarantee an optimal detection of a next-hop forwarder, since no information about the physical distance is considered. Indeed, in a transmission range, the farthest vehicle from a source vehicle should be the most likely next-hop forwarder of the packet as this will yield the highest forward progress. It follows that MOVES technique has to exploit ( $i$ ) the physical dependence by preferring the farthest node, while guaranteeing $(i i)$ the social degree. Finally, it is worth to notice that Eq. (9) shows the time dependence of MOVES forwarding probability assignment function, coming from the social degree $s_{i}(t)$. It is then expected to observe a variation of the forwarding probability assignment function based on the width of the time windows $\Delta T$, which affects the social degree variability.

Figure 3 depicts the dependance of MOVES forwarding probability assignment function by both the distance from a Tx vehicle and the social degree 


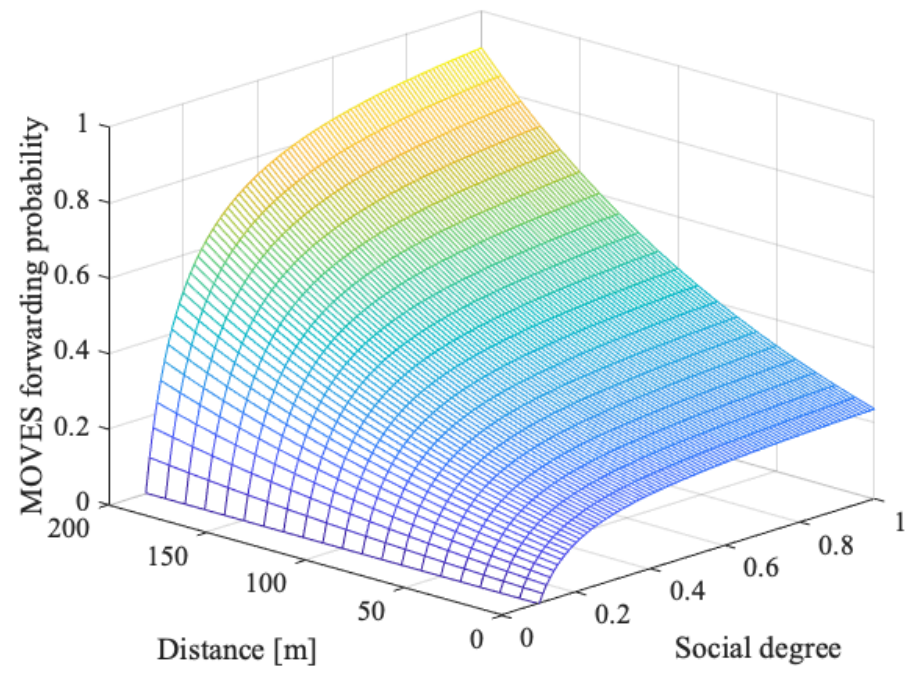

Figure 3: MOVES forwarding probability of the $i$-th vehicle at distance $d_{i}[\mathrm{~m}]$ (i.e., $d_{i} \leq z$ $[\mathrm{m}]$ ) and social degree $s_{i}$ (i.e., $s_{m} \leq s \leq 1$, with $s_{m}=0.1$ ). In particular, we assumed $z=200$ $[\mathrm{m}], \rho=0.02[\mathrm{veh} / \mathrm{m}], c=3$, and $\alpha=2$.

$s_{i}$. Here, we assume static values of the social degree, while in Section 4 we 1 will present simulated results for different values of the time window $\Delta T$. In 2 Figure 3 we observe that MOVES forwarding probability exists for $s_{i} \geq s_{m}$, and is higher when $s_{i} \rightarrow 1$ and $d_{i} \rightarrow z[\mathrm{~m}]$. In practice, MOVES takes into account 4 both $(i)$ the physical factor by means of inter-vehicle distance, then supporting $\quad 5$ the farthest vehicle, and $(i i)$ the social aspect of vehicles. The farthest and most social vehicle is the preferable next-hop node. In conclusion, the selection of a next-hop forwarder occurs on the basis of the highest MOVES assignment function within a transmission range.

Differently from SCARF [12], MOVES considers the social aspect of the $i$-th vehicle as a single event i.e., the probability that there is no other neighbor- ${ }_{11}$ ing vehicle with a higher social degree, which is statistically independent from 12 the event related to the physical factors. Indeed, MOVES forwarding probabil- $\quad{ }_{13}$ ity assignment function considers two statistically independent events that are $\quad 14$ related to $(i)$ physical factors, by means of the inter-vehicle distance and $(i i) \quad 15$ 


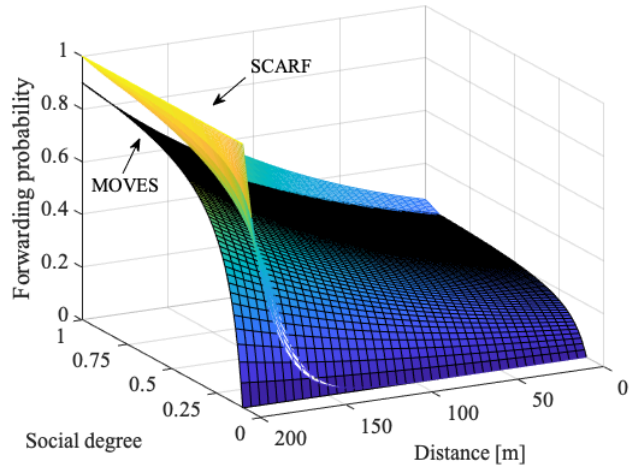

$(a)$

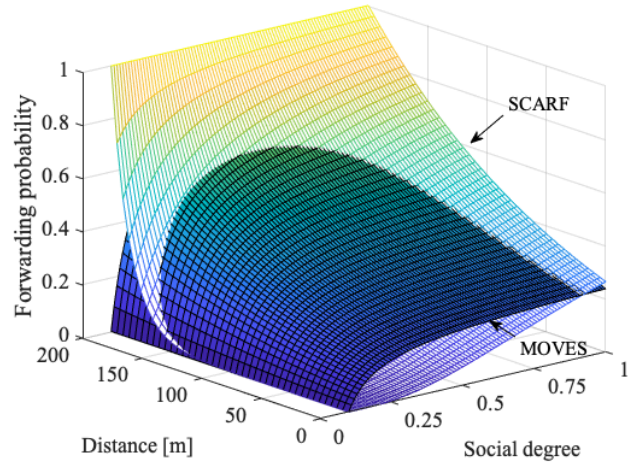

(b)

Figure 4: Comparison of MOVES and SCARF forwarding probabilities versus the distance and the social degree. (a) MOVES shows lower probability than SCARF for high social degree and high distance, while (b) SCARF probability is lower when the social degree and the distance are lower.

social factors, by means of the social degree of the $i$-th node. On the other side, SCARF technique is based on a single event only i.e., the probability that there is no neighboring vehicle at distance $\left(z-d_{i}\right)[\mathrm{m}]$ with a social degree $s_{i}$. No comparison to other vehicles in terms of social aspect is provided.

To better understand the behavior of MOVES, as compared to SCARF, we 6 observe the plots in Figure 4, where the two curves overlap to each other in 7 particular areas. Specifically, for high social degree and high distance, MOVES 8 forwarding probability results lower than SCARF (see Figure $4(a)$ ). Indeed, 9 MOVES considers social aspects and physical ones associated to two separated 10 events, and then cannot reach the maximum value of probability. For lower 11 social degree and distance values, MOVES presents higher trend than SCARF, 12 as depicted in Figure $4(b)$. Again, this is due to the exponential nature of SCARF forwarding probability assignment function, which reaches high values only for higher social degree and distances. On the other side, MOVES essentially represents a power law with an exponential cutoff, where the exponential decay second term of Eq. (9) overwhelms the power-law behavior at very large 


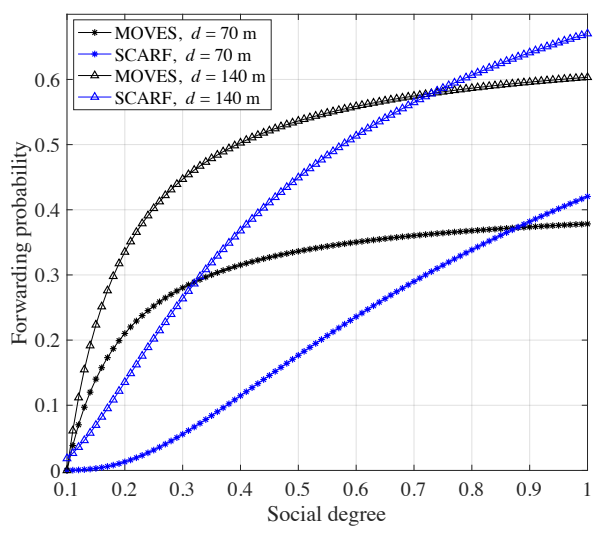

$(a)$

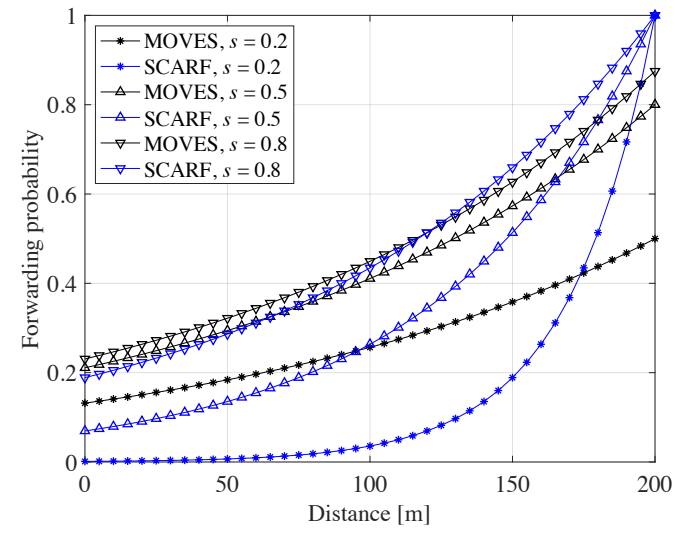

(b)

Figure 5: Comparison of MOVES and SCARF forwarding probabilities $(a)$ for different values of distance $d_{i}[\mathrm{~m}]$ (i.e., $d_{i} \leq z[\mathrm{~m}]$ ), versus the social degree, and (b) for different values of social degree $s_{i}$, versus the distance. We assumed $z=200[\mathrm{~m}], \rho=0.02[\mathrm{veh} / \mathrm{m}], c=3$, $s_{m}=0.1$ and $\alpha=2$.

values of the social degree.

Finally, we observe that SCARF and MOVES alternate their trends, so 2 that the first looks more efficient in detecting those vehicles laying at long 3 distances and with high social degree, while the latter assigns higher probability 4 to those vehicles at lower distance and with lower social degree. MOVES shows 5 a smooth trend for $s_{i} \rightarrow 1$ and $d_{i} \rightarrow z$, while SCARF forwarding probability 6 reaches the maximum value very fast, due to the exponential factor of the inter- 7 vehicle distance. For instance, for high values of distance $\left(e . g ., d_{i}=190[\mathrm{~m}] \quad 8\right.$ with $z=200[\mathrm{~m}])$ and low social degree $\left(e . g ., s_{i}=0.2\right)$, SCARF forwarding 9 probability is 0.716 , while for MOVES it is 0.467 . In general, for maximum 10 distance and independently from the social degree, SCARF shows its maximum ${ }_{11}$ probability, while MOVES reaches its highest value only if the vehicle is the 12 farthest and most social in the transmission range. For low distances and high 13 social degree, both the approaches tend to assigning low probability values (e.g., ${ }^{14}$ for $s_{i}=1$ and $d_{i}=10[\mathrm{~m}]$, SCARF probability is 0.281, while for MOVES it is ${ }_{15}$ 


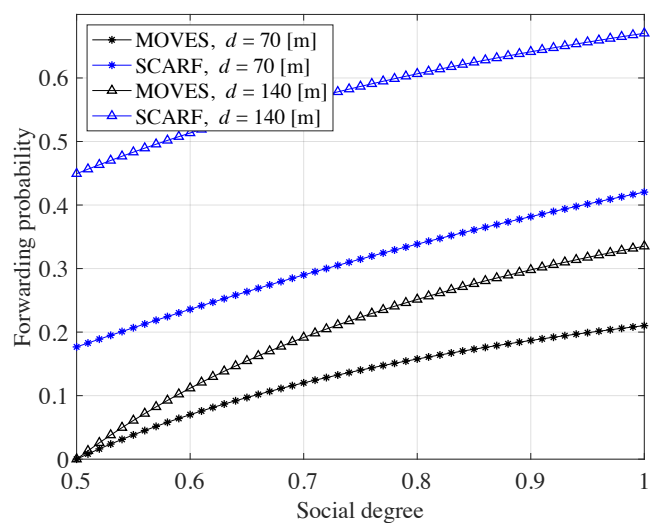

(a)

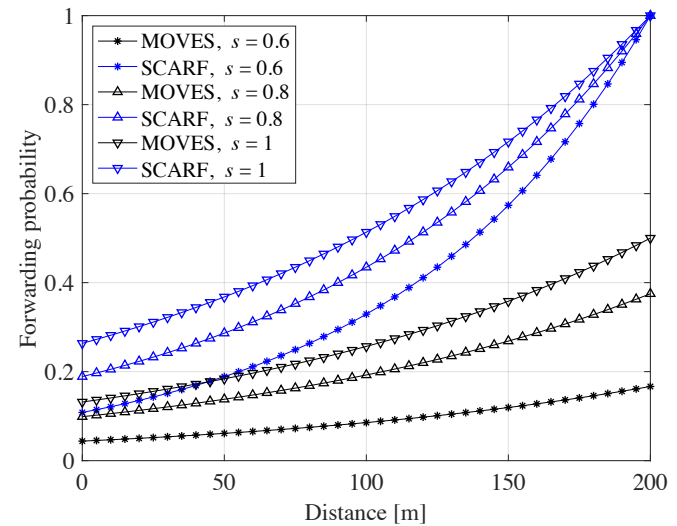

(b)

Figure 6: Comparison of MOVES and SCARF forwarding probability in case of $s_{m}=0.5$, versus $(a)$ social degree, and $(b)$ distance. We assumed $z=200[\mathrm{~m}], \rho=0.02[\mathrm{veh} / \mathrm{m}], c=3$, and $\alpha=2$.

$0.253)$

A more detailed description about the comparison between SCARF and MOVES is presented in Figure $5(a)$, which depicts the two curves for fixed values of distance i.e., $d_{i}=[70,140] \mathrm{m}$, versus the social degree. We observe that MOVES curve starts with slightly higher probability values than SCARF, and then the trend varies slowly for increasing social degree. On the other side, SCARF assigns very low values of forwarding probability due to low social degree, but then increases fast till overcoming MOVES for very high social degree. MOVES trend is smoother, and looks like saturating for increasing social degree.

In Figure $5(b)$, similar considerations can be applied by comparing MOVES and SCARF for fixed values of social degree i.e., $s_{i}=[0.2,0.5,0.8]$, versus the distance. It is easy to observe that SCARF shows different curves that 14 reach the maximum probability faster than MOVES, which in contrast shows 15 a maximum probability that is always lower than SCARF. Again, we observe that MOVES overcomes SCARF in particular ranges due to its faster increase 


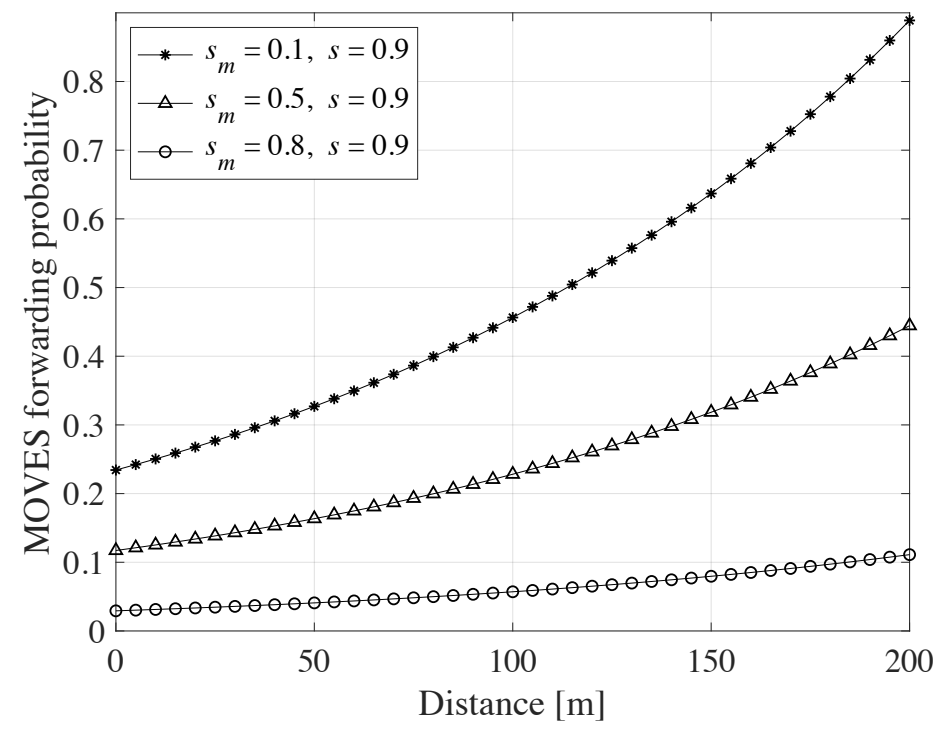

Figure 7: Dependence of MOVES forwarding probability on the minimum social degree parameter $s_{m}$.

at the beginning, followed by a slower trend for increasing distances. Finally, 1 while SCARF presents different curves for different values of social degree, for 2 increasing social degree MOVES shows very close probability values. 3

In all previous results, MOVES has been evaluated for $s_{m}=0.1$. However, $\quad 4$ it is observed from Eq. (9) that MOVES forwarding probability strictly depends 5 on the minimum value of social degree i.e., $s_{m}$, as for $s_{i}<s_{m}$ the probability is $\quad 6$ null. In Figure 6 we depict MOVES trend in case of $s_{m}=0.5$, as compared to 7 SCARF. Notice that MOVES provides a forwarding probability only if $s_{i} \geq s_{m}$. $\quad 8$ This aspect represents a quality requirement since only those nodes exhibiting 9 a social degree higher than a given threshold are eligible as potential next-hop 10 forwarders. The minimum social degree value that a vehicle should exhibit lim- ${ }_{11}$ its the selection of potential forwarders i.e., the higher (lower) the minimum 12 social degree the lower (higher) the MOVES forwarding probability (see Fig- ${ }_{13}$ ure 7). This allows the use of MOVES in applications needing higher quality ${ }_{14}$ requirements (i.e., comfort and interactive entertainment), while SCARF does ${ }_{15}$ 


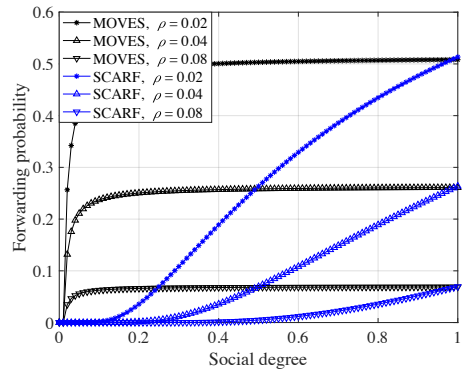

(a)

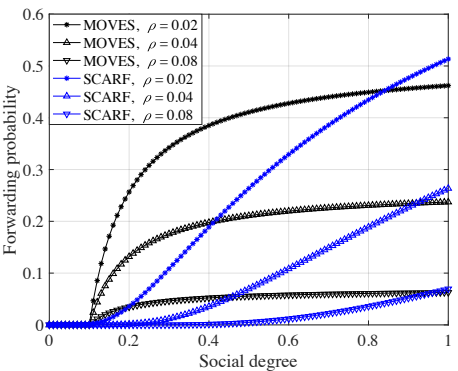

(b)

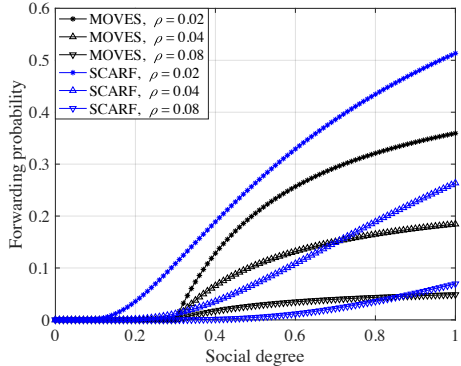

(c)

Figure 8: MOVES forwarding probability versus the social degree, for different values of vehicular density $\rho$, in case of $(a) s_{m}=0.01,(b) s_{m}=0.1$ and $(c) s_{m}=0.3$.

not introduce any constraint on the social degree i.e., potentially all the vehicles have no social degree limitation and can become a next-hop forwarder.

Finally, Figure 8 depicts MOVES and SCARF forwarding probabilities behavior for different values of vehicular density i.e., $\rho=[0.02,0.04,0.08] \mathrm{veh} / \mathrm{m}$, computed for a vehicle at distance $d=100 \mathrm{~m}$ from a source node (again, $d<z$ 6 and $z=200 \mathrm{~m}$ ). Also, MOVES has been evaluated in case of different values 7 of the minimum social degree $s_{m}$ i.e., $s_{m}=[0.01,0.1,0.3]$. It can be observed 8 (see Figure $8(c)$ ) that for increasing $s_{m}$, the area of existence of the MOVES 9 forwarding probability shifts to increasing social degree, with a reduced trend.

Differently, in case of very low minimum value of social degree $s_{m}$ as in Figure 8 (a), the behavior of MOVES forwarding probability saturates very quickly, with higher (lower) trend in case of low (high) vehicular density.

\subsection{Average Number of Forwarders}

In order to assess the propagation efficiency of MOVES forwarding technique, we are interested in the expected number of hops needed to transmit a packet from a source to a destination node. Let us consider the scenario where an information packet propagates from a source node to a destination one. We assume $N_{z}$ as the number of potential forwarder vehicles in the transmission range $[0, z][\mathrm{m}]$ of a source vehicle; each of them can be elected as a social 


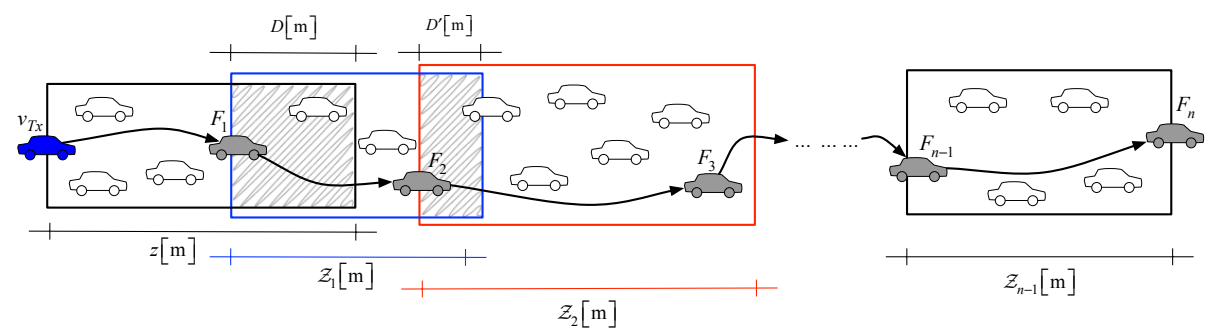

Figure 9: Schematic model of the propagation range in case of multi-hop scenario. A source vehicle (blue vehicle) will select the next-hop forwarder $F_{1}$ (gray vehicle) within its transmission range $z[\mathrm{~m}]$. Notice that the transmission ranges are randomly distributed in the range $[z / 2,3 z / 2][\mathrm{m}]$.

next-hop forwarder, according to Eq. (9).

As known $[12,44]$, the number of potential forwarders $N_{z}$ within the source's transmission range can be assumed as a random variable, according to a Poissonstatistical distribution with parameter $\rho z$, whose Probability Density Function $(\mathrm{PDF})$ is:

$$
f_{N_{z}}(n)=\operatorname{Pr}\left\{N_{z}=n\right\}=\frac{(\rho z)^{n} e^{-\rho z}}{n !},
$$

and then it follows that the expected value of $N_{z}$ can be written as

$$
\begin{aligned}
\mathbb{E}\left\{N_{z}\right\} & =\int_{1}^{N_{z}} n f_{N_{z}}(n) d n \\
& =\int_{1}^{N_{z}} \frac{n(\rho z)^{n} e^{-\rho z}}{n !} d n \\
& =\int_{1}^{N_{z}} e^{-\rho z} \frac{(\rho z)^{n}}{\Gamma(n)} d n,
\end{aligned}
$$

where $\Gamma(n)$ is the Gamma function defined as

$$
\Gamma(n)=\int_{0}^{\infty} e^{-t} t^{n-1} d t .
$$

The solution of Eq. (11) can be computed numerically for different values of 8 $N_{z}$, which correspond to different values of $\rho$, as $N_{z}=\rho z$. Eq. (11) considers $\quad 9$ the number of forwarders within one transmission range i.e., $z[\mathrm{~m}]$. However, 10 we are also interested in the average number of forwarders in a multi-hop prop- ${ }_{11}$ agation. For this aim, we consider the scenario as depicted in Figure 9, where ${ }_{12}$ 


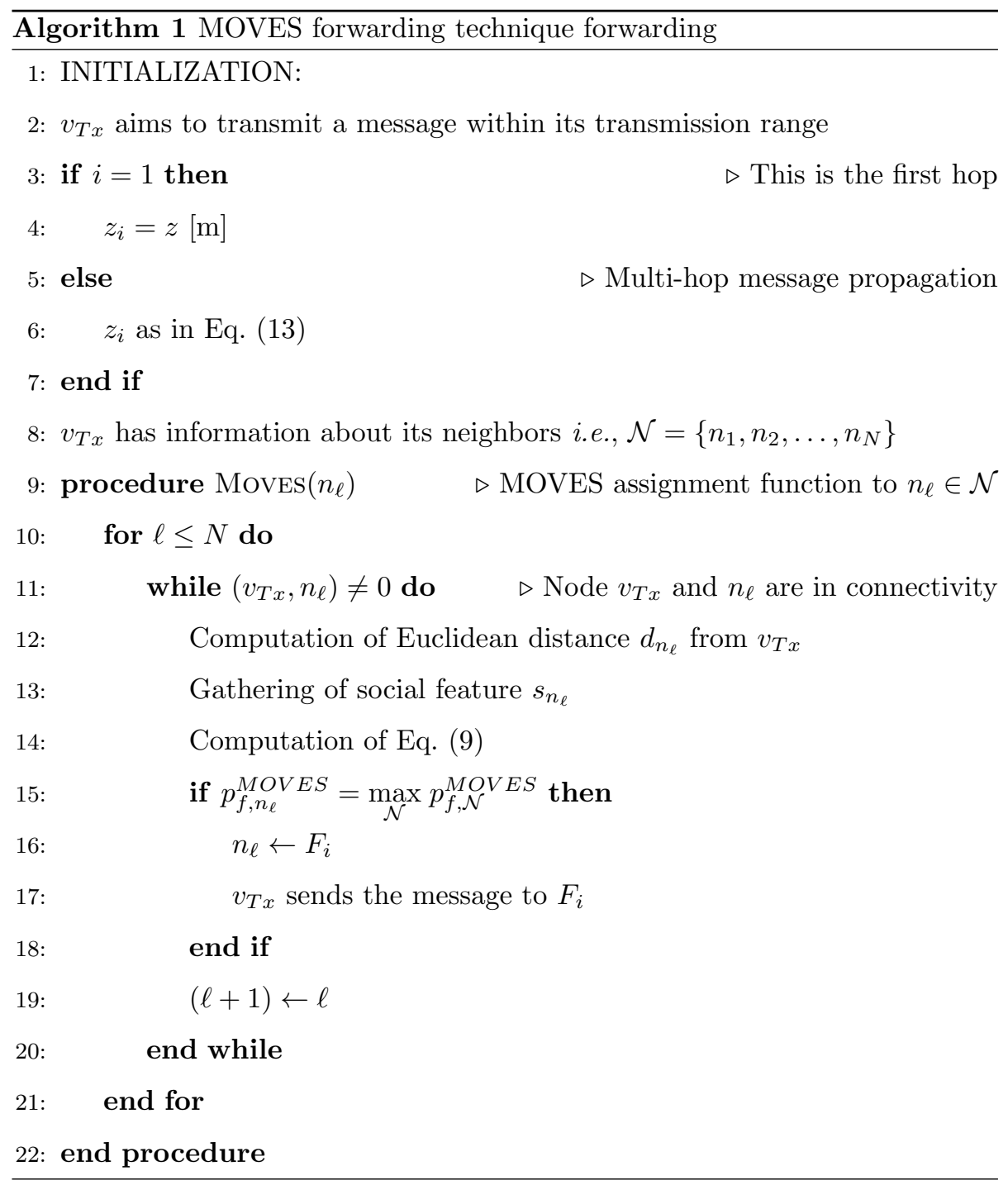


we assume that the transmission ranges of the next-hop forwarders (i.e., $F_{n} \quad 1$ vehicles, with $n \in \mathbb{Z}^{+}$as the number of hops) are randomly distributed in the 2 interval $[z / 2,3 z / 2][\mathrm{m}]$. This assumption can represent a vehicular scenario with 3 fading effect and noisy channel. Also, the inter-distance of vehicles within the 4 transmission ranges is randomly distributed.

Algorithm 1 describes the main objective of MOVES technique. A source vehicle $v_{T x}$ (i.e., blue vehicle) aims to send a message within its transmission range and selects the forwarder node $\left(i . e ., F_{1}\right)$ from the set of all its neighbors $\mathcal{N}=\left\{n_{1}, n_{2}, \ldots, n_{N}\right\}$, showing the highest MOVES forwarding probability. The selection of node $F_{1}$ is based on both its physical distance and social feature. Such information is obtained by assuming an edge computing architecture, as already described in [12]. In the next-hop transmission, vehicle $F_{1}$ will select vehicle $F_{2}$ within the transmission range $z^{\prime}[\mathrm{m}]$. Notice that vehicle $F_{1}$ will seek for potential forwarders those vehicles that are in the area $\left(z^{\prime}-D\right)[\mathrm{m}]$, where $D[\mathrm{~m}]$ is the overlapping area with the transmission range of previous hop. This is due in order to avoid the selection of next-hop forwarders that also belong to previous transmission range. Finally, in the next-hop transmission, vehicle $F_{2}$ will seek for potential forwarders in the area $\left(z^{\prime \prime}-D^{\prime}\right)$, where $z^{\prime \prime}[\mathrm{m}]$ is $F_{2} \quad{ }_{18}$ transmission range and $D^{\prime}[\mathrm{m}]$ is the overlapping area with $z^{\prime}[\mathrm{m}]$ transmission $\quad 19$ range. According to such criterium, $F_{3}$ will be selected as next-hop forwarder. $\quad 20$ In general, for a multi-hop propagation, the transmission range of the $i$-th node $\quad 21$ where the next-hop forwarder will be selected is computed as:

$$
z_{i}=\mathcal{Z}_{i}-\mathcal{Z}_{i-1}+d_{i, i-1}
$$

where $\mathcal{Z}_{i}[\mathrm{~m}]$ is whole transmission range of the $i$-th forwarder and $d_{i, i-1}[\mathrm{~m}]$ is $\quad{ }_{23}$ the inter-distance between two consecutive forwarders.

Figure 10 depicts the average number of forwarders for different values of $\quad 25$ vehicular density $\rho$, in case of single and multiple transmissions, i.e., from one 26 to four hops, respectively. Specifically, in Figure 10 we assume that the trans- $\quad 27$ mission range in one-hop scenario is $z=200 \mathrm{~m}$, and then the number of nodes $\quad 28$ within this range is $N_{z}=\rho z$, with $\rho=[0.02,0.17][\mathrm{veh} / \mathrm{m}]$. As expected, the 29 


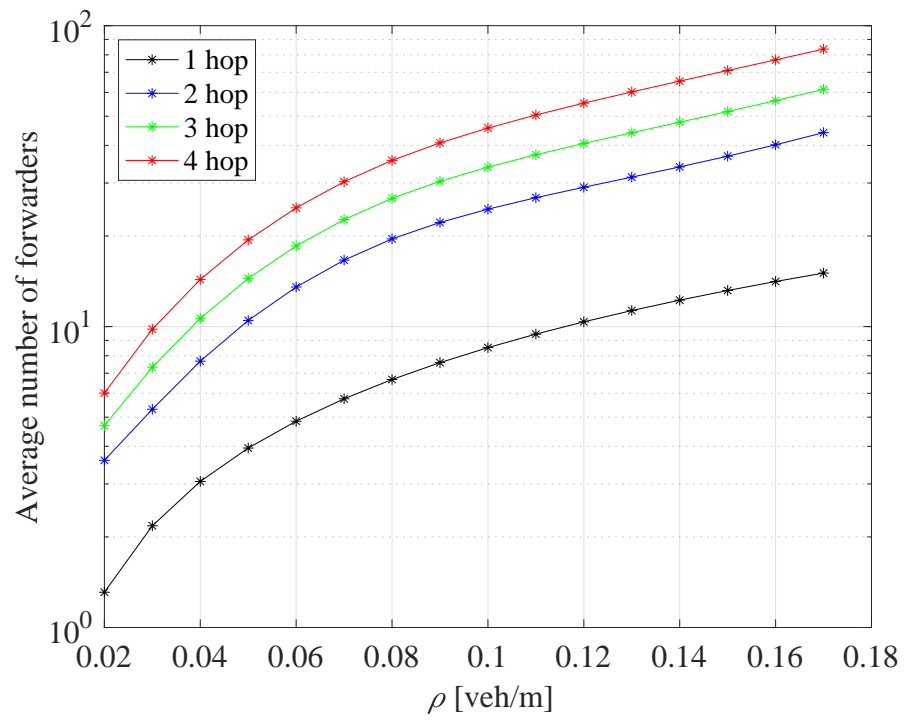

Figure 10: Average number of forwarders for different vehicular densities $\rho$ and number of hops.

average number of forwarders increases with the number of transmissions, as well as with the vehicular density $\rho$.

$3 \quad$ Now, by considering the PDF of the social degree i.e., $f_{S}(\cdot)$, is Pareto4 distributed [45] with $\alpha$ as the exponent of the power law distribution and $s_{m}$ as

5 the minimum value of the social degree, we can compute the expected value of 6 the social degree distribution as:

$$
\begin{aligned}
\mathbb{E}\{S\} & =\int_{s_{m}}^{s} s f_{S}(s) d s \\
& =\int_{s_{m}}^{s} s \frac{\alpha-1}{s_{m}}\left(\frac{s}{s_{m}}\right)^{-\alpha} d s .
\end{aligned}
$$

7 Eq. (14) can be exploited in order to compute the expected value of the number 8 of social forwarders in 1 hop transmission i.e., $S F_{z}$, as

$$
\mathbb{E}\left\{S F_{z}\right\}=\mathbb{E}\left\{N_{z}\right\} \cdot \mathbb{E}\{S\}
$$

9 with $z[\mathrm{~m}]$ as the transmission range of first hop. In case of multi-hop propaga- 


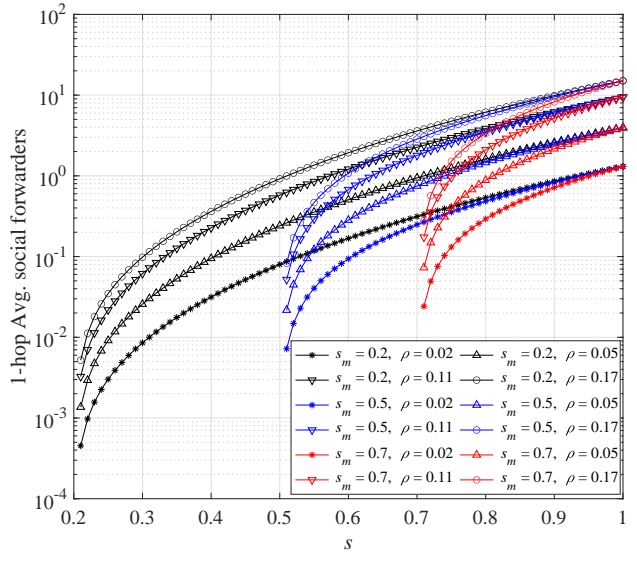

$(a)$

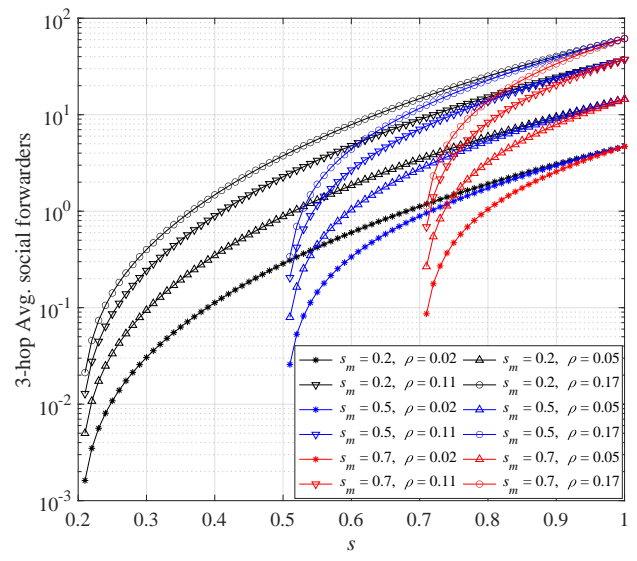

$(c)$

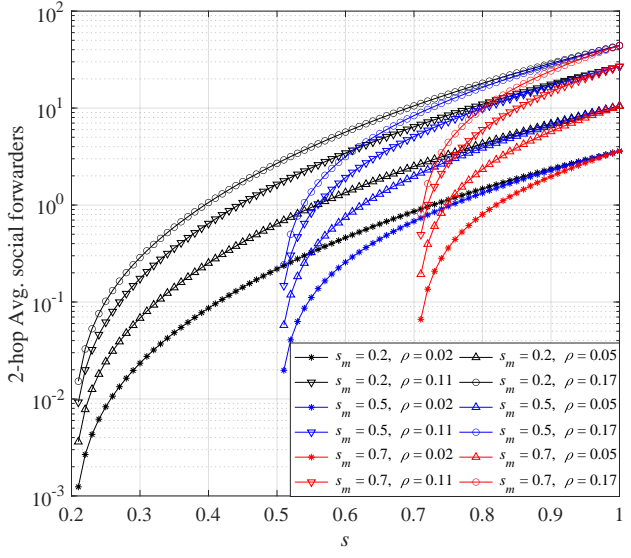

(b)

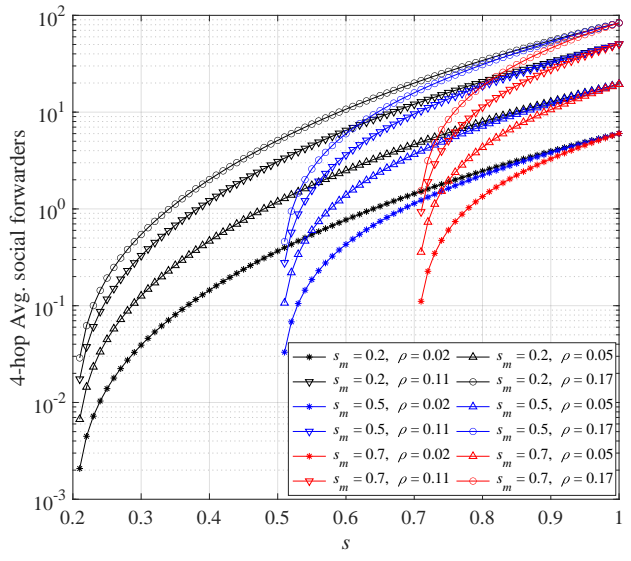

$(d)$

Figure 11: Average number of social forwarders vs the social degree, for different vehicular density $\rho$ and minimum value of social degree $s_{m}$, in case of (a) 1 hop, (b) 2 hops, (c) 3 hops and $(d) 4$ hops. Here, we considered $\alpha=2$. 
1 tion, Eq. (15) becomes

$$
\mathbb{E}\left\{S F_{z_{i}}\right\}=\mathbb{E}\left\{N_{z_{i}}\right\} \cdot \mathbb{E}\{S\}
$$

where $z_{i}$ is defined in Eq. (13).

In Figure 11, the average number of potential social forwarders has been evaluated by considering the impact of $(i)$ different vehicular densities (i.e., $\rho$ ) and $(i i)$ the minimum social degree $\left(\right.$ i.e., $\left.s_{m}\right)$. We estimate the average number of potential social forwarders for different number of hops. Indeed, in order to the $i$-th node to be eligible as social forwarder within a transmission range, its corresponding social degree has to be higher than a given minimum social degree (i.e., $s_{i} \geq s_{m}$ ). These figures give us important information on the average number of potential forwarders that we can find in subsequent hops, when we consider not only the purely physical conditions of the nodes, but also their social propensity to re-send data. As expected, the average number of potential forwarders increases with higher hops, since we consider "cumulative" forwarders. The interesting aspect of these curves is that even though the minimum social threshold value is different (i.e., $s_{m}=[0.2,0.5,0.7]$ ), for the same density value, the curves converge towards the same value of forwarders for $s \rightarrow 1$. Finally, it is worth to highlight that MOVES, after detecting potential social forwarders within a transmission range, will select one of them based on a probabilistic comparison.

\section{Simulation results}

Performance of MOVES forwarding technique has been assessed via extended simulations, and also compared to other existing routing techniques. Two simulation scenarios have been considered i.e., (i) San Francisco [46] and (ii) Rome taxies [47]. The first scenario is simulated by means of real taxi mobility traces from San Francisco, USA, where 533 taxis in the San Francisco Bay Area were monitored by recording their GPS positions over 30 days [46], moving along a grid topology. The second scenario was acquired by an extensive measurement campaign in the city of Rome (Italy), in which 370 taxi cabs working in 
Table 1: Simulation parameters used in San Francisco [46] and Rome taxi [47] scenarios.

\begin{tabular}{l|c|c}
\hline \hline Parameter & San Francisco & Rome taxi \\
\hline Number of nodes & 533 & 370 \\
\hline Number of traces & 11220492 & 449226 \\
\hline Complete trace duration [days] & 30 & 30 \\
\hline Trace frequency monitoring $[\mathrm{s}]$ & 10 & 7 \\
\hline Network range $[\mathrm{m}]$ & {$[10,50]$} & {$[10,50]$} \\
\hline Bandwidth $[\mathrm{MBps}]$ & 10 & 10 \\
\hline Buffer size $[\mathrm{MB}]$ & 50 & 50 \\
\hline Message size $[\mathrm{kB}]$ & {$[100,1000]$} & {$[100,1000]$} \\
\hline \hline
\end{tabular}

the center of Rome, characterized by high congestion streets and following a 1 road topology, reported their positions at every 7 seconds for a period of six 2 months [47]. Table 1 collects the main parameters assumed in the simulation 3 results, which have been obtained in The One simulator [48].

In this experimentation, for both scenarios, we have simulated a group of dif- $\quad 5$ ferent nodes forming a vehicular communication network. Specifically, we based 6 our simulations on Active-DTN [49], i.e., an Opportunistic Network solution 7 that implements the Bundle Protocol and additionally extends it by allowing 8 the messages being communicated to incorporate software and message state 9 for forwarding, delivery, lifetime control and prioritization purposes. Then, we 10 have adapted our enhanced version of The One simulator to include the pro- ${ }_{11}$ posed MOVES technique by implementing the social degree and the forwarding 12 probability assignment function, as defined in Eq. (1) and Eq. (9), respectively. ${ }_{13}$

We first aim to assess that the distribution of the node social degree in real ${ }_{14}$ vehicular trace scenarios shows a power-law trend. Figure 12 and 13 depict the ${ }_{15}$ percentage of social nodes in San Francisco and Rome taxi scenarios, respec- ${ }_{16}$ tively, computed for different values of $r$ (i.e., $r=\Delta T / T$, assuming values in ${ }_{17}$ 


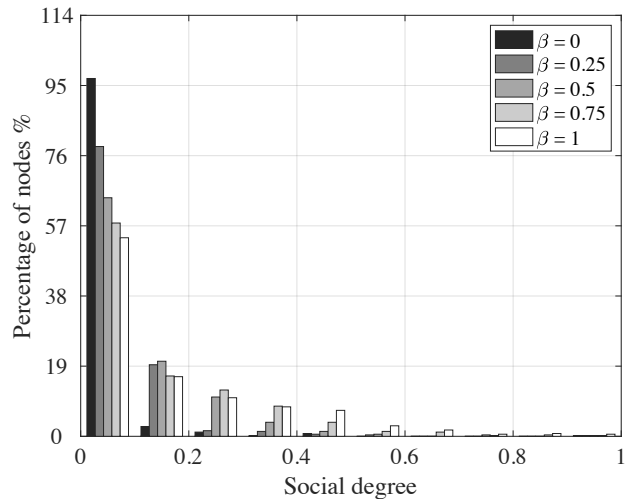

(a)

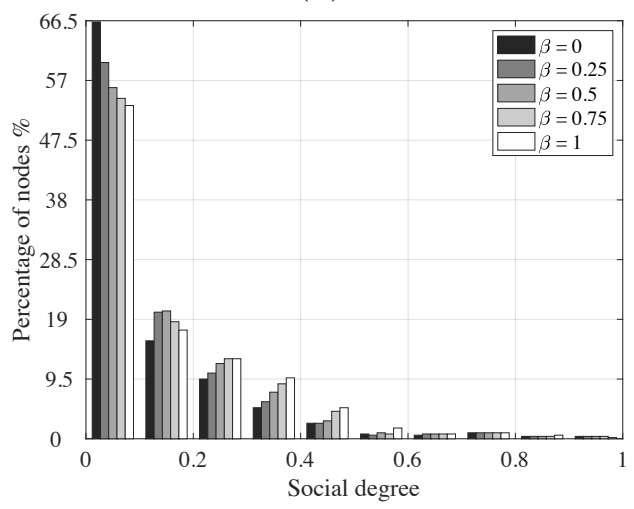

$(c)$

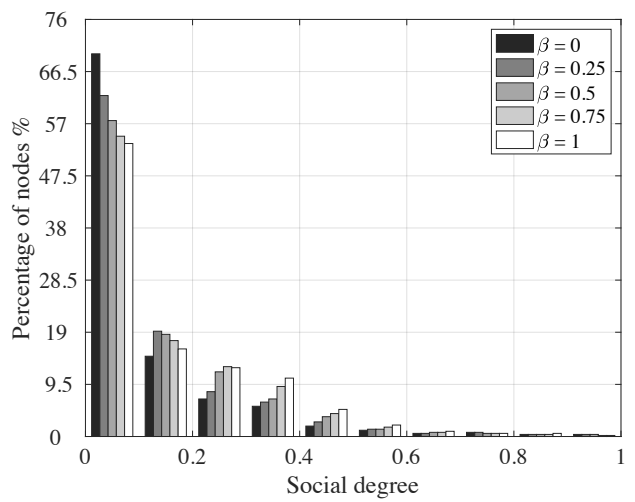

(b)

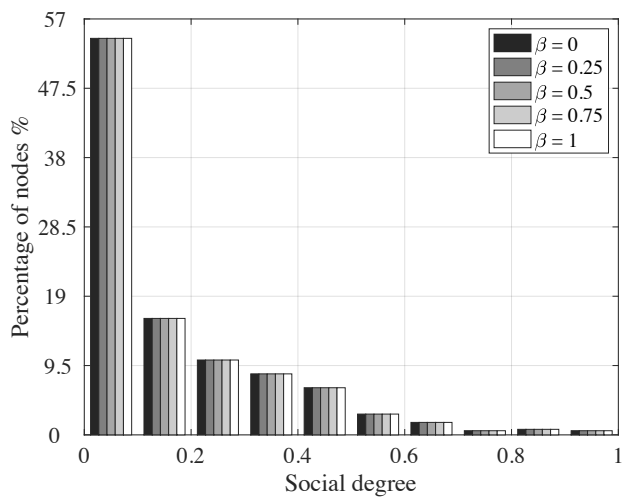

$(d)$

Figure 12: Percentage of nodes in San Francisco scenario, with a given social degree in the range $[0,1]$, in case of $(a) r=\Delta T / T=0.25,(b) r=0.5,(c) r=0.75$, and $(d) r=1$.

1 the range $[0.25,1]$ ), and parameter $\beta$ (i.e., $\beta=[0,1]$ ). As expected, we notice

2 that the social degree is Pareto-distributed, with a large percentage of nodes

3 with a social degree lower than 0.2 . The parameter $\beta$ affects the social degree

4 by opportunely weighting the message and connection coefficients, as expressed

5 in Eq. (2) and (3), respectively. For low values of $\beta$ (i.e., $\beta<0.5$ ), the frequency

6 of nodes with the lowest social degree $(i . e ., s<0.2)$ is bigger when $r$ is smaller

7 or equal to 0.75 . Also, for increasing $r$ we can appreciate a decrease of the

8 percentage of nodes with a given social degree. However, when $r$ is equal to 1 , 


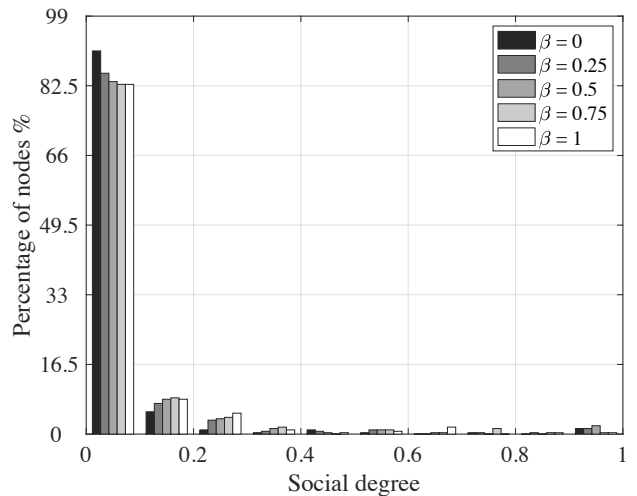

$(a)$

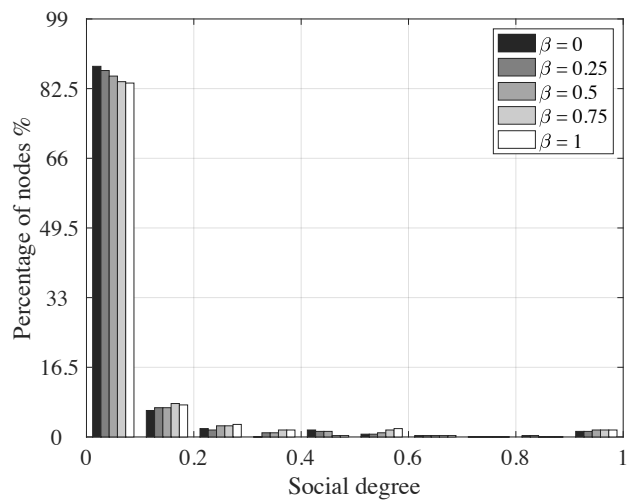

$(c)$

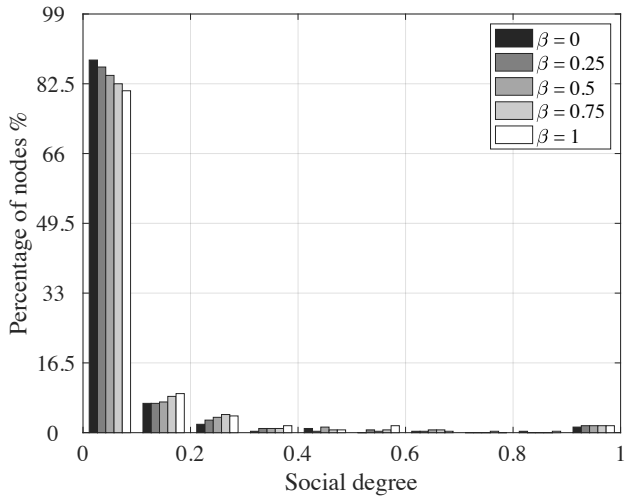

(b)

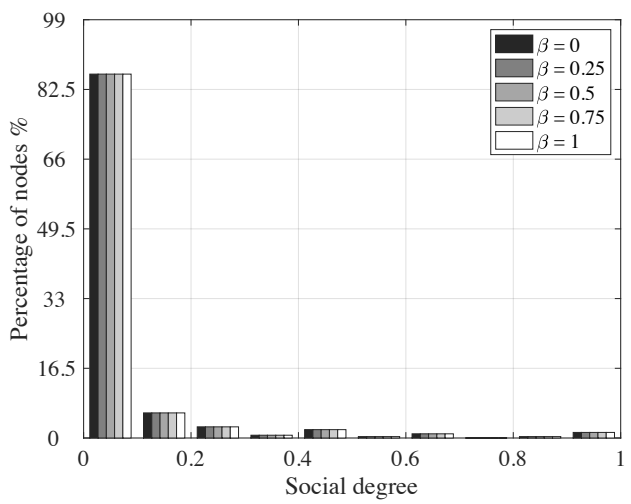

$(d)$

Figure 13: Percentage of nodes in Rome taxi scenario, with a given social degree in the range $[0,1]$, in case of $(a) r=0.25,(b) r=0.5,(c) r=0.75$, and $(d) r=1$.

the social degree is equally distributed, no matter the value of $\beta$.

Next, we focus on the network performance of MOVES approach, as com- 2 pared to three state-of-the-art approaches. Firstly, we compare MOVES with 3 SCARF [19], that is the pillar approach from which MOVES derives. Secondly, 4 we compare MOVES with Epidemic routing [50], where messages are always 5 routed for every contacted node. Finally, we consider an optimal stopping-based 6 approach [20], namely OS, that is a routing protocol where message custodian 7 nodes are limited by carefully selecting their prospective forwarders in terms of 8 


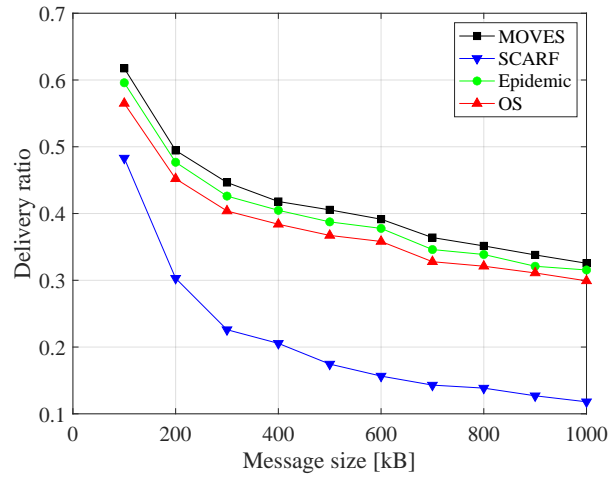

(a)

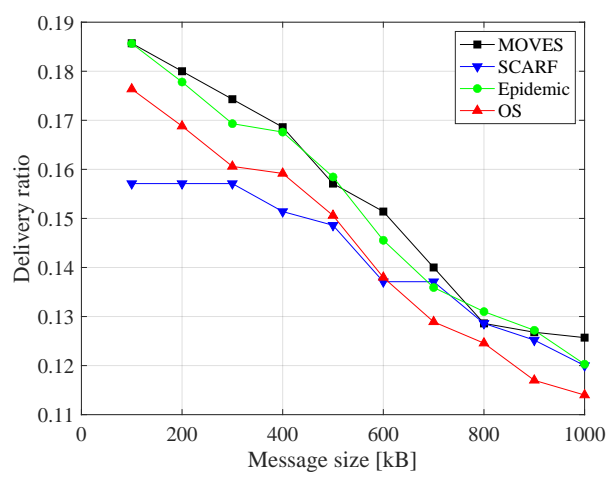

(b)

Figure 14: Comparison of MOVES delivery ratio w.r.t. Epidemic, OS and SCARF techniques, in (a) San Francisco and (b) Rome taxi scenario.

their reliability, network centrality and similarity. OS is based on the Optimal Stopping theory, which allows to select the best message storer, while holding back broad message dissemination until a relay node is met. Specifically, we will investigate $(i)$ the packet delivery ratio, (ii) the overhead, (iii) the number of hops, and (iv) the latency time. As it will be clearer in the following, MOVES behaves better than these proposals, with a higher delivery ratio, lower overhead and latency, and similar number of hops.

Firstly, Figure 14 depicts the delivery ratio i.e., $\mathcal{T}$, defined as the ratio 9 of delivered messages to the number of created messages, for the four studied approaches in case of different scenarios, respectively. In both scenarios, we observe that MOVES shows higher delivery ratio, with a decreasing trend for increasing message sizes. In the best conditions (i.e., a low message size), we get $\mathcal{T}_{\text {MOVES }} \approx 0.6$, while worst results are obtained for larger message sizes, although MOVES reaches always values higher than $\approx 0.35$. We notice that MOVES shows values slightly higher than the Epidemic curve. As a matter, the high replication in Epidemic can flood buffer resources and can have an impact on the delivery ratio. On the other side, in the context of Rome taxi scenario, performance is worst for all four studied routing approaches, as depicted in 


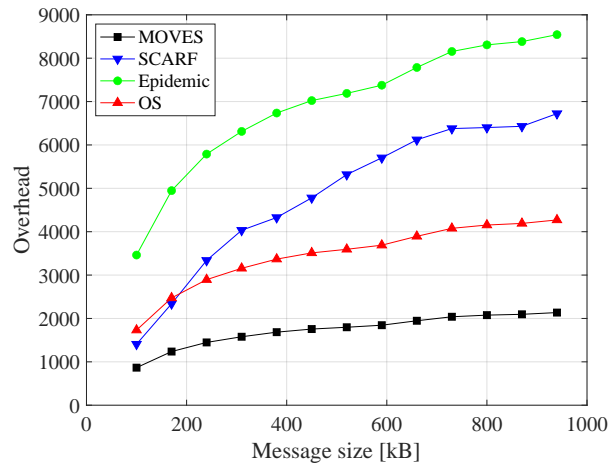

$(a)$

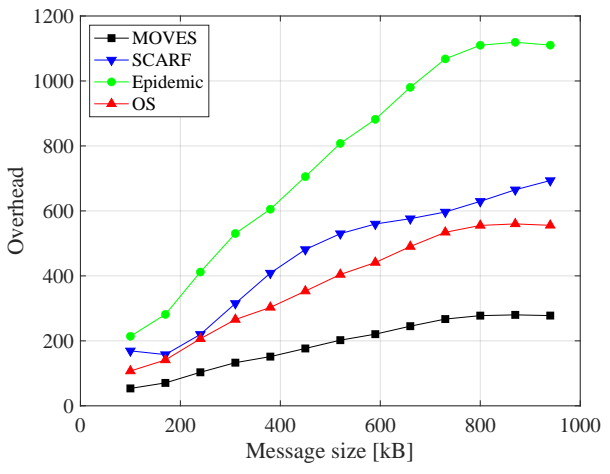

(b)

Figure 15: Comparison of MOVES overhead w.r.t. Epidemic, OS and SCARF techniques in (a) San Francisco and (b) Rome taxi scenario.

Figure $14(b)$. More in detail, we observe that MOVES cannot reach $\approx 0.2$, as 1 well as SCARF does not overcome $\approx 0.16$. Interesting, SCARF slowly decreases 2 for increasing message sizes, and outperforms OS for message size higher than 3 $600 \mathrm{kB}$. This behavior is mainly due to the nature of the simulated scenario, 4 where the percentage of nodes with a social degree higher than 0.2 is around 5 $5 \%$; on the other side, in San Francisco with a higher number of nodes the 6 percentage of nodes with a social degree higher than 0.2 is around $10 \%$, which is twice the value in Rome taxi scenario.

Figure 15 describes the overhead comparison i.e., $\mathcal{O}$, in different scenarios. This is calculated by computing the ratio of the difference between the number of relayed messages i.e., $\ell$, and the number of delivered messages i.e., $\mathcal{D}$, to the number of delivered ones, i.e.

$$
\mathcal{O}=\frac{\ell-\mathcal{D}}{\mathcal{D}}
$$

with $\ell \geq \mathcal{D}$. We observe that MOVES always presents better performance than 13 SCARF, Epidemic and optimal stopping-based approach, with a dynamic that 14 increases slowly. Specifically, in Figure $15(a)$ we have $\mathcal{O}_{\text {MOV ES }} \approx[1000,2000], \quad{ }_{15}$ while highest values are obtained with Epidemic ranging in $\approx[3200,8500]$, with $\quad 16$ 


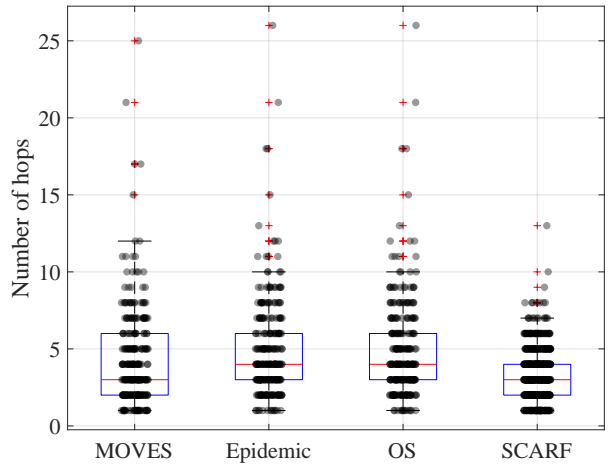

$(a)$

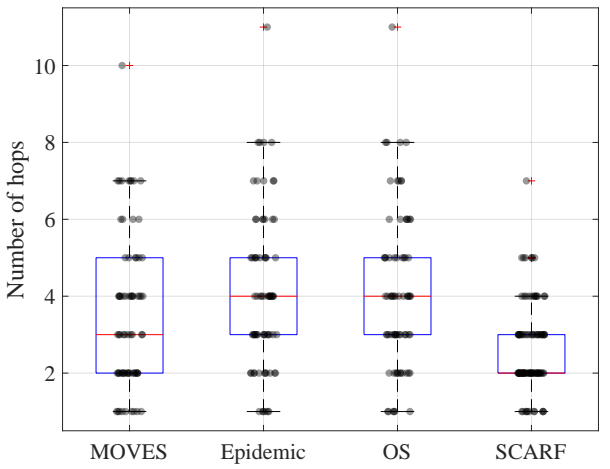

$(b)$

Figure 16: Comparison of MOVES number of hops w.r.t. Epidemic, OS and SCARF techniques in (a) San Francisco and (b) Rome taxi scenario.

message size of $\approx[100,1000] \mathrm{kB}$, respectively. In case of small message size i.e., $100[\mathrm{kB}]$, SCARF shows a value of overhead close to that of MOVES, but then we observe a fast increase of the overhead for increasing message size. Furthermore, in Figure $15(b)$ the trends are similar to those in San Francisco scenario, but the values of overhead are lower of a ten multiplicative factor.

Figure 16 shows the average number of hops that the four approaches perform to disseminate messages in the whole network. This is calculated as the number of forwarders the messages are relayed to before arriving at destination. Due to the different features of San Francisco and Rome taxi scenarios, we observe a higher number of hops in the former scenario as compared to the latter. In case of San Francisco scenario depicted in Figure 16 (a), MOVES and SCARF exhibit similar results with a slightly increase experienced with Epidemic and OS. Similar considerations apply to the number of hops observed in Rome taxi scenario, as shown in Figure $16(b)$.

Finally, in Figure 17, the latency time is analysed. This is calculated as the difference between the message delivery time and the message creation time.

The plotbox depicts, for each message generated in the simulations, the average latency time and the interquartile range between a first quartile (i.e., $Q 1=25 \%$ ) 


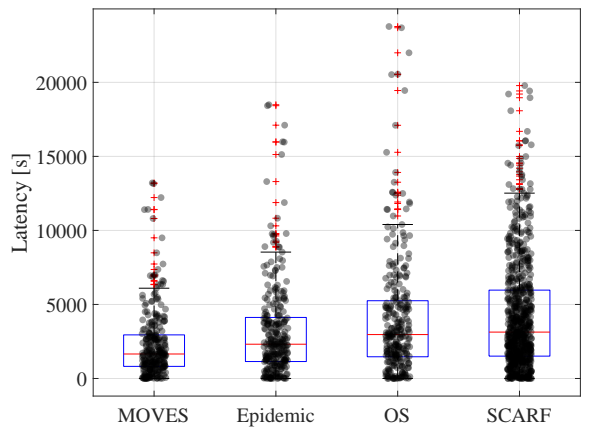

(a)

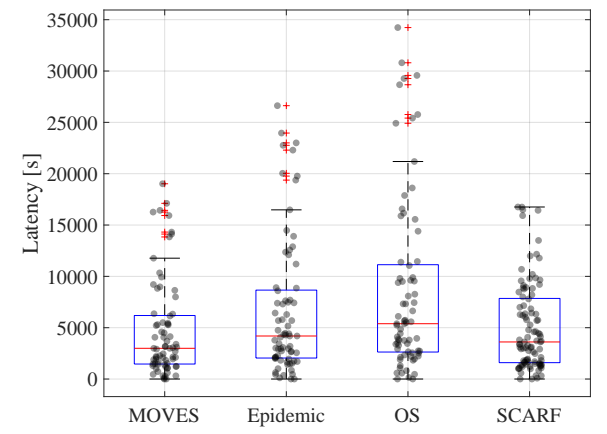

(b)

Figure 17: Comparison of MOVES latency time w.r.t. Epidemic, OS and SCARF techniques in (a) San Francisco and (b) Rome taxi scenario.

and the third quartile (i.e., $Q 3=75 \%$ ). As it can be seen, our proposed MOVES technique performs better in terms of the latency time when comparing it to the other three techniques, for both two scenarios.

To summarize, we can conclude that MOVES shows better network performance as it achieves a higher delivery ratio, lower overhead and latency. This is due to the MOVES forwarding probability assignment function that relies on the selection of less social nodes as next-hop forwarders. This aspect does not represent a disadvantage of the proposed technique, but allows the enhancement of network performance, as compared to Epidemic, optimal stopping-based routing and SCARF approaches.

\section{Conclusions}

Social relations and ties in vehicular contexts have become very popular in 12 the last few years and attracted the interest of the research community. It ${ }_{13}$ has been demonstrated that their integration in the forwarding mechanisms 14 can highly impact on the performance of the network. This integration of social 15 components in vehicular networks is certainly related to the enormous success of 16 the online social networks, and new paradigms such as Vehicular Social Networks 
have been introduced. VSNs are formed on-the-fly and normally social relations are considered ephemeral, but it has been also highlighted that specific patterns can emerge and would be important of considering them.

Based on this rationale, in this paper we have proposed a MemOry-based VEhicular Social (MOVES) forwarding approach. MOVES is based both on a time-varying social degree and physical parameters for the selection of the next-hop forwarder. The specific feature of MOVES resides into the evaluation of the social degree of a node in the past and in the present observations, by effectively exploiting the "social memory" of a node. In order to assess the behavior of MOVES, we have compared it to other existing related techniques i.e., Epidemic, OS, and SCARF, by considering real vehicular data. The performance evaluation has been dealt in terms of delivery ratio, overhead, latency and computational cost, namely number of hops. We have observed that MOVES outperforms other approaches, in terms of delivery ratio, overhead and latency, by demonstrating the higher accuracy of the "social past" inclusion in the data forwarding. The computational cost is also comparable to that from SCARF, but MOVES exhibits higher performance. As next step for improving the effectiveness of the data forwarding, we aim to integrate a prediction algorithm in the MOVES mechanism, for taking into account the quality of a physical link.

\section{References}

[1] V. Arnaboldi, A. Guazzini, A. Passarella, Egocentric Online Social Networks: Analysis of Key Features and Prediction of Tie Strength in Facebook, Computer Communications 36 (03 2013). doi:10.1016/j.comcom.2013.03.003.

[2] A. M. Vegni, V. Loscrí, A Survey on Vehicular Social Networks, IEEE Comm. Surveys \& Tutorials 17 (4) (2015) 2397-2419.

[3] K. M. Alam, M. Saini, A. E. Saddik, Toward Social Internet of Vehicles: Concept, Architecture, and Applications, IEEE Access 3 (2015) 343-357. doi:10.1109/ACCESS.2015.2416657. 
[4] B. Zhang, R. Tian, C. Li, Content dissemination and routing for vehicular 1 social networks: A networking perspective, IEEE Wireless Communications 2 27 (2) (2020) 118-126. doi:10.1109/MWC.001.1900225.

[5] J. Niu, J. Fan, L. Wang, M. Stojinenovic, K-hop Centrality Metric for 4 Identifying Influential Spreaders in Dynamic Large-scale Social Networks, 5 in: 2014 IEEE Global Communications Conference, 2014, pp. 2954-2959. 6 doi:10.1109/GLOCOM.2014.7037257.

[6] Y. Igarashi, T. Miyazaki, A DTN routing algorithm adopting the "Com- 8 munity" and "Centrality" parameters used in social networks, in: 2018 9 International Conference on Information Networking (ICOIN), 2018, pp. 10 211-216. doi:10.1109/ICOIN.2018.8343112.

[7] H. Li, Centrality Analysis of Online Social Network Big Data, in: $2018{ }_{12}$ IEEE 3rd International Conference on Big Data Analysis (ICBDA), 2018, 13 pp. 38-42. doi:10.1109/ICBDA.2018.8367648. ${ }_{14}$

[8] B. Zhang, R. Tian, C. Li, Content Dissemination and Routing for Vehicular ${ }_{15}$ Social Networks: A Networking Perspective, IEEE Wireless Communica- ${ }_{16}$ tions (2019) 1-9doi:10.1109/MWC.001.1900225.

[9] H. Zhou, M. Ruan, C. Zhu, V. C. M. Leung, S. Xu, C. Huang, ${ }_{18}$ A Time-Ordered Aggregation Model-Based Centrality Metric for ${ }_{19}$ Mobile Social Networks, IEEE Access 6 (2018) 25588-25599. 20 doi:10.1109/ACCESS.2018.2831247. ${ }_{21}^{21}$

[10] A. Chakraborty, V. B. S., B. S. Manoj, Analytical Identification of Anchor 22 Nodes in a Small-World Network, IEEE Communications Letters 20 (6) ${ }_{23}$ (2016) 1215-1218. doi:10.1109/LCOMM.2016.2554117. ${ }_{24}$

[11] Y. Guo, Z. Liu, Y. Zeng, R. Wang, J. Ma, Preserving Privacy for ${ }_{25}$ Hubs and Links in Social Networks, in: 2018 International Conference ${ }_{26}$ on Networking and Network Applications (NaNA), 2018, pp. 263-269. ${ }_{27}$ doi:10.1109/NANA.2018.8648740. 28 
[12] A. M. Vegni, C. Souza, V. Loscrí, E. Hernández-Orallo, P. Manzoni, Data

2 Transmissions Using Hub Nodes in Vehicular Social Networks, IEEE Transactions on Mobile Computing 19 (7) (2020) 1570-1585.

[13] V. Raghavan, G. Ver Steeg, A. Galstyan, A. G. Tartakovsky, Modeling Temporal Activity Patterns in Dynamic Social Networks, IEEE Transactions on Computational Social Systems 1 (1) (2014) 89-107. doi:10.1109/TCSS.2014.2307453.

[14] S. Zeng, J. Zhang, Analyse Social Influence on Student Motivation based on Social Activity Network, in: 2016 IEEE International Conference on Service Operations and Logistics, and Informatics (SOLI), 2016, pp. 133138. doi:10.1109/SOLI.2016.7551675.

[15] Z. Li, C. Wang, S. Yang, C. Jiang, X. Li, LASS: Local-Activity and Social-Similarity Based Data Forwarding in Mobile Social Networks, IEEE Transactions on Parallel and Distributed Systems 26 (1) (2015) 174-184. doi:10.1109/TPDS.2014.2308200.

[16] G. Wang, L. Zhang, Z. Yang, X. Li, Socialite: Social Activity Mining and Friend Auto-labeling, in: 2018 IEEE 37th International Performance Computing and Communications Conference (IPCCC), 2018, pp. 1-8. doi:10.1109/PCCC.2018.8710834.

[17] A. Peleshchyshyn, V. Vus, O. Markovets, S. Albota, Identifying Specific Roles of Users of Social Networks and Their Influence Methods, in: 2018 IEEE 13th International Scientific and Technical Conference on Computer Sciences and Information Technologies (CSIT), Vol. 2, 2018, pp. 39-42. doi:10.1109/STC-CSIT.2018.8526635.

[18] A. Aylani, N. Goyal, Community detection in Social Network based on Users Social Activities, in: 2017 International Conference on I-SMAC (IoT in Social, Mobile, Analytics and Cloud) (I-SMAC), 2017, pp. 625-628. doi:10.1109/I-SMAC.2017.8058254. 
[19] A. M. Vegni, V. Loscrí, R. Petrolo, SCARF: A SoCial-Aware Reliable 1 Forwarding Technique for Vehicular Communications, in: Proceedings of 2 the 3rd Workshop on Experiences with the Design and Implementation of 3 Smart Objects, SMARTOBJECTS '17, ACM, Snowbird, Utah, USA, 2017, 4 pp. 1-6. doi:10.1145/3127502.3127512.

URL http://doi .acm.org/10.1145/3127502.3127512

[20] C. Borrego and J. Borrell and S. Robles, Efficient broadcast in opportunistic 7 networks using optimal stopping theory, Ad Hoc Networks 88 (2019) 5-17. 8

[21] N. Jabeur, S. Zeadally, B. Sayed, Mobile Social Networking Applications, 9 ACM Communications 56 (3) (2013) 71-79. 10

[22] X. Liang, R. Lu, X. Lin, X. Shen, Security and Privacy in Mobile Social ${ }_{11}$ Networks, Springer, 2013.

[23] N. Montavont, S. Hachana, L. Loiseau, Opportunistic Social Network ${ }_{13}$ Experiments, in: 2009 International Conference on Advanced Infor- ${ }_{14}$ mation Networking and Applications Workshops, 2009, pp. 850-855. ${ }_{15}$ doi:10.1109/WAINA.2009.134.

[24] A. Socievole, F. D. Rango, A. Caputo, Opportunistic Mobile Social ${ }_{17}$ Networks: From Mobility and Facebook friendships to structural analysis 18 of user social behavior, Elsevier Computer Communications 87 (2016) 1 - 19 18. doi:https://doi.org/10.1016/j.comcom.2016.04.025. 20 URL http://www.sciencedirect.com/science/article/pii/ ${ }_{21}$ S0140366416301803 22

[25] L. Tang, Z. Duan, Y. Zhu, Data Dissemination in Service Discovery for ${ }^{23}$ Vehicular ad hoc Networks: a Survey, IET Intelligent Transport Systems 24 12 (10) (2018) 1189-1200. 25

[26] A. Papadimitriou, D. Katsaros, Y. Manolopoulos, Social Network Anal- ${ }_{26}$ ysis and its Applications in Wireless Sensor and Vehicular Networks, in: ${ }_{27}$ International Conference on e-Democracy, Springer, 2009, pp. 411-420. 28 
[27] M. S. Frigau, Social-based forwarding in multi-channel vehicular networks, in: 2015 IEEE Symposium on Computers and Communication (ISCC), 2015, pp. 166-173. doi:10.1109/ISCC.2015.7405511.

[28] S. A. Bitaghsir and S. Kashipazha and A. Dadlani and A. Khonsari, Socialaware Mobile Road Side Unit for Content Distribution in Vehicular Social Networks, in: 2019 IEEE Symposium on Computers and Communications (ISCC), 2019, pp. 1-6. doi:10.1109/ISCC47284.2019.8969669.

[29] E. Pagani, L. Valerio, G. P. Rossi, Weak Social Ties improve Content Delivery in Behavior-aware Opportunistic Networks, Ad Hoc Networks 25 (2015) 314 - 329, new Research Challenges in Mobile, Opportunistic and Delay-Tolerant Networks Energy-Aware Data Centers: Architecture, Infrastructure, and Communication. doi:https://doi.org/10.1016/j.adhoc.2014.07.005.

URL http://www.sciencedirect.com/science/article/pii/ S157087051400136X

[30] F. Pianese, X. An, F. Kawsar, H. Ishizuka, Discovering and Predicting user Routines by Differential Analysis of Social Network Traces, 2013 IEEE 14th International Symposium on A World of Wireless, Mobile and Multimedia Networks (WoWMoM) (2013) 1-9.

[31] H. Gong, L. Yu, X. Zhang, Social Contribution-Based Routing Protocol for Vehicular Network with Selfish Nodes, International Journal of Distributed Sensor Networks 10 (4) (2014) 753024. doi:10.1155/2014/753024.

[32] F. Gai, J. Zhang, P. Zhu, X. Jiang, Trust on the Ratee: A Trust Management System for Social Internet of Vehicles, Wireless Communications and Mobile Computing 2017 (2017).

[33] N. Basta, A. ElNahas, H. P. Grossmann, S. Abdennadher, A framework for social tie strength inference in vehicular social networks, in: 2019 Wireless Days (WD), 2019, pp. 1-8. doi:10.1109/WD.2019.8734218. 
[34] L. Yao and Y. Wang and X. Wang and G. WU, Cooperative Caching in 1 Vehicular Content Centric Network Based on Social Attributes and Mo- 2 bility, IEEE Transactions on Mobile Computing 20 (2) (2021) 391-402. 3 doi:10.1109/TMC.2019.2944829.

[35] N. Ullah and X. Kong and L. Lin and M. Alrashoud and A. Tolba and F. 5 Xia, Real-time dissemination of emergency warning messages in 5G enabled 6 selfish vehicular social networks, Computer Networks 182 (2020) $107482 . \quad$ 7

[36] Z. Li, J. Bi, C. Borrego, Exploiting Temporal and Spatial Regularities for 8 Content Dissemination in Opportunistic Social Network, Hindawi, Wireless 9 Communications and Mobile Computing 2019 (2019) $1-16 . \quad 10$

[37] M. Motani, V. Srinivasan, P. S. Nuggehalli, PeopleNet: Engineering a ${ }_{11}$ Wireless Virtual Social Network, in: Proceedings of the 11th Annual In- ${ }_{12}$ ternational Conference on Mobile Computing and Networking, MobiCom ${ }_{13}$ '05, Association for Computing Machinery, New York, NY, USA, 2005, p. ${ }_{14}$ 243-257. doi:10.1145/1080829.1080855. URL https://doi.org/10.1145/1080829.1080855

[38] H. Lenando and M. Alrfaay , EpSoc: Social-Based Epidemic-Based Routing $\quad{ }_{17}$ Protocol in Opportunistic Mobile Social Network, Hindawi Mobile Infor- ${ }_{18}$ mation Systems 2018 (2018). doi:10.1155/2018/6462826. 19

[39] U. Lee, J. Lee, J. Park, M. Gerla, FleaNet: A Virtual Market Place on 20 Vehicular Networks, IEEE Transactions on Vehicular Technology 59 (1) ${ }_{21}$ (2010) 344-355. doi:10.1109/TVT.2009.2030892. 22

[40] R. Tomar, H. G. Sastry, M. Prateek, A Novel Protocol for Information 23 Dissemination in Vehicular Networks, in: C.-H. Hsu, S. Kallel, K.-C. Lan, ${ }_{24}$ Z. Zheng (Eds.), Internet of Vehicles. Technologies and Services Toward 25 Smart Cities, Springer International Publishing, Cham, 2020, pp. 1-14. ${ }_{26}$

[41] Z. Zhou, C. Gao, C. Xu, Y. Zhang, S. Mumtaz, J. Rodriguez, Social Big- ${ }_{27}$ 
1 Data-Based Content Dissemination in Internet of Vehicles, IEEE Transac-

2 tions on Industrial Informatics 14 (2018) 768-777.

3 [42] F. Mezghani, R. Dhaou, M. N. Lima, A.-L. Beylot, Content Dissemina4 tion in Vehicular Social Networks: Taxonomy and User Satisfaction, IEEE

$5 \quad$ Communications Magazine 52 (2014) 34-40.

6 [43] M. Newman, Power laws, Pareto distributions and Zipf's law, Contempo$7 \quad$ rary Physics 46 (5) (2005) 323-351. doi:10.1080/00107510500052444.

8 [44] S. Abuelenin, Moment Analysis of Highway-Traffic Clearance Distribution, IEEE Transactions on Intelligent Transportation Systems 16 (2015) 2543 2550. doi:10.1109/TITS.2015.2412117. Effective is to Look at a Vehicular Network under a Social Perception?, in: Proc. of IEEE 9th International Conference on Wireless and Mobile Computing, Networking and Communications (WiMob), 2013, pp. 154159.

[46] M. Piorkowski, N. Sarafijanovic-Djukic, M. Grossglauser, Crawdad data set epfl/mobility (v. 2009-02-24) (2009).

[47] R. Amici, M. Bonola, L. Bracciale, A. Rabuffi, P. Loreti, G. Bianchi, Performance Assessment of an Epidemic Protocol in VANET Using Real Traces, in: Proc. of International Conference on Selected Topics in Mobile and Wireless Networking (MoWNeT), Vol. 40, 2014, pp. 92-99. doi:10.1016/j.procs.2014.10.035.

[48] A. Keränen, J. Ott, T. Kärkkäinen, The ONE Simulator for DTN Protocol Evaluation, in: Proceedings of the 2nd International Conference on Simulation Tools and Techniques, Simutools ?09, ICST (Institute for Computer Sciences, Social-Informatics and Telecommunications Engineering), Brussels, BEL, 2009. doi:10.4108/ICST.SIMUTOOLS2009.5674. URL https://doi.org/10.4108/ICST.SIMUTOOLS2009.5674 
[49] C. Borrego, S. Robles, A. Fabregues, A. Sánchez-Carmona, A mobile code 1 bundle extension for application-defined routing in delay and disruption 2 tolerant networking, Computer Networks 87 (2015) 59-77.

[50] X. Zhang, G. Neglia, J. Kurose, D. Towsley, Performance modeling of epi- ${ }_{4}$ demic routing, Computer Networks 51 (10) (2007) 2867-2891. 